Document downloaded from:

http://hdl.handle.net/10251/161859

This paper must be cited as:

Cuenca-Gotor, VP.; Sans-Tresserras, JȦ.; Gomis, O.; Mujica, A.; Radescu, S.; Muñoz, A.; Rodríguez-Hernández, P.... (2020). Orpiment under compression: metavalent bonding at high pressure. Physical Chemistry Chemical Physics. 22(6):3352-3369.

https://doi.org/10.1039/c9cp06298j

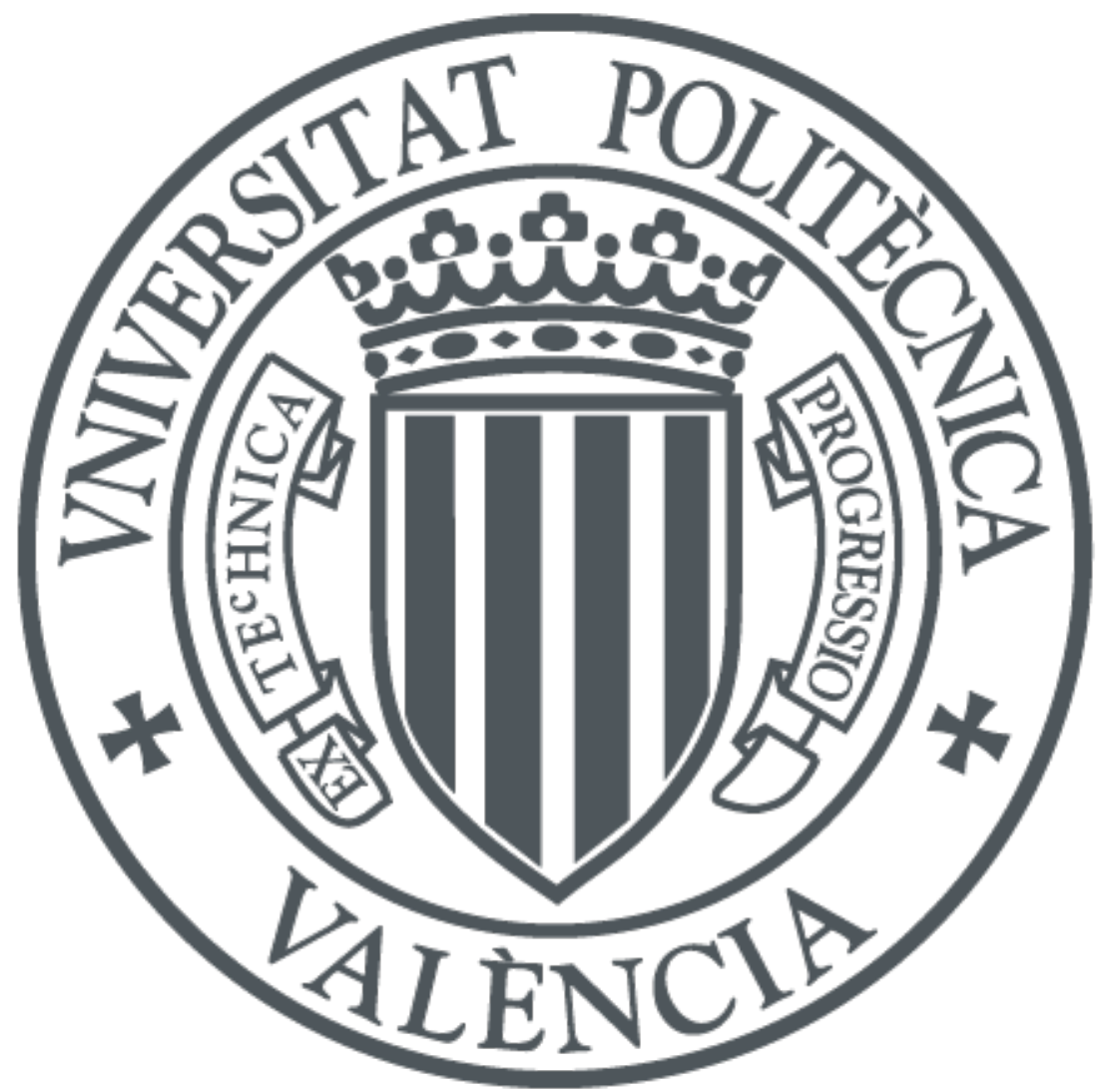

The final publication is available at

https://doi.org/10.1039/c9cp06298j

Copyright The Royal Society of Chemistry

Additional Information 


\section{Orpiment under compression: metavalent bonding at high pressure}

Received 00th January 20xx, Accepted 00th January 20xx DOI: $10.1039 / \times 0 \times x 00000 x$
Vanesa Paula Cuenca-Gotor, ${ }^{a}$ Juan Ángel Sans, $,{ }^{a},{ }^{*}$ Oscar Gomis, ${ }^{b}$ Andres Mujica, ${ }^{c}$ Silvana Radescu, ${ }^{c}$ Alfonso Muñoz, ${ }^{c}$ Plácida Rodríguez-Hernández, ${ }^{c}$ Estelina Lora da Silva, ${ }^{a}$ Catalin Popescu, ${ }^{d}$ Jordi Ibañez, ${ }^{\mathrm{e}}$ Rosario Vilaplana, ${ }^{\mathrm{b}}$ and Francisco Javier Manjón ${ }^{\mathrm{a},{ }^{*}}$

\begin{abstract}
We report a joint experimental and theoretical study of the structural, vibrational, and electronic properties of layered monoclinic arsenic sulfide crystal $\left(\alpha-\mathrm{As}_{2} \mathrm{~S}_{3}\right)$, aka mineral orpiment, under compression. X-ray diffraction and Raman scattering measurements performed in orpiment samples at high pressure and combined with ab initio calculations have allowed us to determine the equation of state and the tentative assignment of the symmetry of many Raman-active modes of orpiment. From our results, we conclude that no first-order phase transition occurs up to $25 \mathrm{GPa}$ at room temperature; however, compression leads to an isostructural phase transition above $20 \mathrm{GPa}$. In fact, As coordination increases from threefold at room pressure to more than fivefold above $20 \mathrm{GPa}$. This increase in coordination can be understood as the transformation from a solid with covalent bonding to a solid with metavalent bonding at high pressure, which results in a progressive decrease of the electronic and optical bandgap, an increase of the dielectric tensor components and Born effective charges, and a considerable softening of many high-frequency optical modes with increasing pressure. Moreover, we propose that the formation of metavalent bonding at high pressures may also explain the behavior of other group-15 sesquichalcogenides under compression. In fact, our results suggest that group-15 sesquichalcogenides either show metavalent bonding at room pressure or undergo a transition from p-type covalent bonding at room pressure towards metavalent bonding at high pressure, as a preceding phase towards metallic bonding at very high pressure.
\end{abstract}

\section{A Introduction}

Arsenic sulfide $\left(\mathrm{As}_{2} \mathrm{~S}_{3}\right)$ and in particular the monoclinic polymorph $\left(\alpha-A s_{2} S_{3}\right)$, aka mineral orpiment, is one of the ores of As together with minerals realgar $\left(\alpha-\mathrm{As}_{4} \mathrm{~S}_{4}\right)$ and arsenopyrite (FeAsS) [1]. In particular, orpiment and realgar have been known since ancient times, with realgar being used as an orange-red pigment and orpiment as a gold-like pigment, hence his mineral name orpiment (Aurum pigmentum in Latin) [2]. Due to the high chemical stability of $\alpha-\mathrm{As}_{2} \mathrm{~S}_{3}$, the canary yellow or King's yellow pigment was obtained from molten orpiment and has been extensively used in papyrus and objects of ancient Egypt that date back to 3.1 millennia BC [3-5]. Additionally, $\mathrm{As}_{2} \mathrm{~S}_{3}$ crystals and glasses have been used as drugs to treat different illnesses, such as prophylactic diseases, asthma, tuberculosis or diabetes, and were also prescribed as antiseptics

a. Instituto de Diseño para la Fabricación y Producción Automatizada, MALTA Consolider Team, Universitat Politècnica de València, 46022 Valencia (Spain).

b. Centro de Tecnologías Físicas, MALTA Consolider Team, Universitat Politècnica de València, 46022 Valencia (Spain).

c. Departamento de Física, Instituto de Materiales y Nanotecnología, MALTA Consolider Team, Universidad de La Laguna, 38200 San Cristóbal de La Laguna (Spain).

d. ALBA-CELLS, MALTA Consolider Team, 08290 Cerdanyola, Barcelona (Spain).

e. Institute of Earth Sciences Jaume Almera, MALTA Consolider Team, CSIC, 08028 Barcelona (Spain).

Electronic Supplementary Information (ESI) available: [details of any supplementary information available should be included here]. See DOI: 10.1039/x0xx00000x and sedative by Aristotle and Hippocrates (IV century BC). Moreover, orpiment has been used since ancient times in traditional Chinese medicine [6] until nowadays, when arsenic sesquisulfide has proved to exhibit positive effects in cancer therapy [7]. Finally, it must be stressed that in the last decades arsenic sulfides have been investigated for different applications in photonics and non-linear optics since they possess high values of the refractive index, high IR transparency, high optical non-linearity, stability to crystallization, the possibility to be modified by a femtosecond laser irradiation, and an inertness to environment $[8,9]$.

$\mathrm{As}_{2} \mathrm{~S}_{3}$ usually crystallizes in the monoclinic $\alpha$ polymorph [space group (SG) $P 2_{1} / n$ or $P 2_{1} / c$, No. 14] [10,11]; although a triclinic dimorph of orpiment, called anorpiment [SG $P \overline{1}$, No. 2] was recently discovered and approved as a new mineral by the International Mineralogical Association [12]. The crystalline structure of the monoclinic $\alpha$ phase (see Figure 1) is composed by corrugated or zigzag layers piled up mainly along the $b$ axis (see Figure 1(a)), which are linked by weak van der Waals (vdW) forces [13].

The properties of arsenic sulfides have been extensively studied, mainly for melts and glasses, because $\mathrm{As}_{2} \mathrm{~S}_{3}$ ranks first among chalcogenides from the viewpoint of the production of amorphous and glass-like industrial materials. However, many properties of their crystalline phases, orpiment, and anorpiment, are not yet well known, likely due to the complex 
(a)

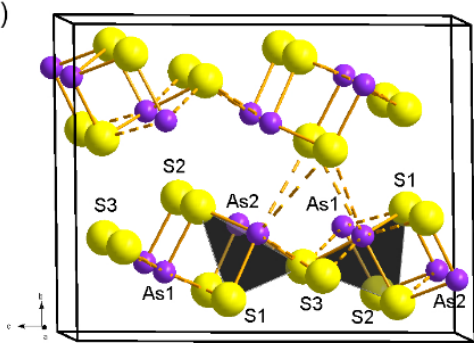

(b)

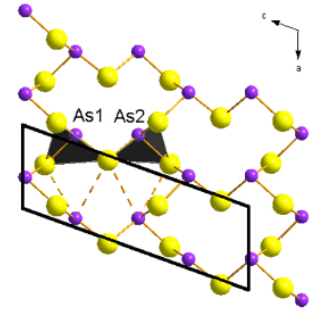

(c)

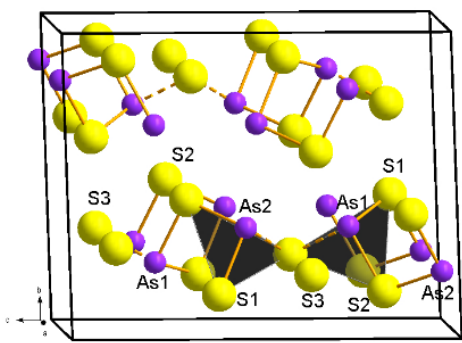

(d)

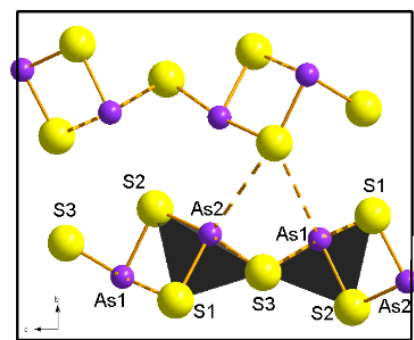

(e)

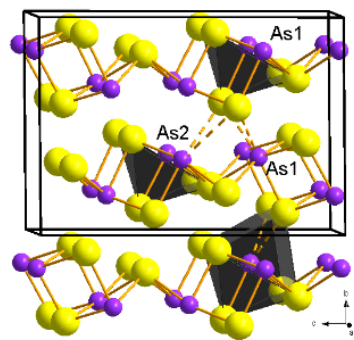

Figure 1. $\alpha-\mathrm{As}_{2} \mathrm{~S}_{3}$ structure at room pressure. Big yellow and small purple spheres correspond to $\mathrm{S}$ and $\mathrm{As}$ atoms, respectively. (a) 3D structure. All solid lines correspond to bonds of similar length ( $2.3 \AA$ ) and dashed lines correspond to longer As-S distances (> $3.0 \AA$ A). (b) Structure of the layer in the ac plane showing the heart-shaped rings. The upper part shows the $\mathrm{AsS}_{3}$ pyramids due to the short bonds that result in the threefold coordination of As and twofold coordination of $\mathrm{S}$ at room pressure. Inside the cell edges we show the long intralayer bonds that would contribute to fivefold coordination of As above 25 GPa. (c) Structure viewed as AsS spiral chains (in As1-S1-As2-S2 sequence) joined by inter-chain S3 atoms. Only short bonds are displayed. Solid (dashed) lines correspond to intra (inter)-chain bonds. (d) Projection of the $\alpha-\mathrm{As}_{2} \mathrm{~S}_{3}$ structure onto the $b c$ plane. (e) $3 \mathrm{D}$ structure of $A s_{2} \mathrm{~S}_{3}$ at $30 \mathrm{GPa}$, showing polyhedral units with fivefold coordination of As1 and As2 atoms (up and middle) and with 5+2 coordination for As1 atoms (down).

monoclinic and triclinic crystalline structures of these compounds. For instance, a definitive symmetry assignment of the vibrational modes of orpiment is still under debate [14] despite a number of papers reporting Raman scattering (RS) measurements, as well as infrared (IR) measurements, since the advent of laser-based Raman spectroscopy [14-29].

In this context, the pressure is a valuable tool to study the properties of materials and several high-pressure (HP) studies have been performed in glassy $A s_{2} S_{3}$ and $\alpha-A s_{2} S_{3}$ in order to help us to understand their properties. In particular, results obtained from HP optical absorption, HP x-ray absorption spectroscopy (XAS), HP Raman scattering (RS) measurements, HP electrical measurements and from a few $a b$ initio

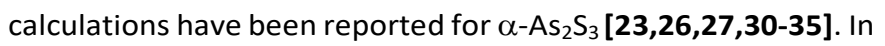
particular, a HP and high-temperature (HT) polymorph of orpiment $\left(\alpha-A s_{2} S_{3}\right)$, whose structure has not been resolved yet, has been found [34]. This is not surprising because little is also known about the structure of orpiment and its behavior under compression. It is only known that the remarkable high packing of layers in orpiment is responsible for its high stability under pressure as suggested by previous experiments. Besides, HPXAS measurements suggested some structural changes above $10 \mathrm{GPa}$ and an increase in As coordination above $30 \mathrm{GPa}$. On the other hand, recent electrical measurements and calculations suggest an isostructural phase transition (IPT) around $25 \mathrm{GPa}$ [33].

To verify these hypotheses, it would be desirable to perform HP $x$-ray diffraction (XRD) measurements on $\alpha-A s_{2} S_{3}$ at room temperature in order to obtain the equation of state of orpiment and explore the origin of the changes proposed in the literature. Such measurements will allow us to investigate the possible appearance of HP phases and its relationship mainly with those of other sesquioxides and sesquichalcogenides. On the other hand, it will be important to perform new HP-RS measurements in order to correlate the structural and vibrational changes in orpiment and to aid in the assignment of the symmetries and the origin of the different vibrational modes with the help of $a b$ initio calculations. All these measurements and calculations would allow us to understand the behavior of orpiment under compression.

For all the mentioned reasons, we report in this work a joint experimental and theoretical study of the structural, vibrational, and electronic properties of $\alpha-\mathrm{As}_{2} \mathrm{~S}_{3}$ under compression at room temperature by means of powder HP-XRD and HP-RS measurements in orpiment, where our experimental results are complemented with theoretical $a b$ initio totalenergy, lattice-dynamics and electronic band structure calculations.

Our structural results show a continuous compression of orpiment up to $25 \mathrm{GPa}$ with a large decrease of the interlayer distance, coherent with the loss of the vdW character of the dominating forces in this region. A progressive increase of As coordination with compression, changing from threefold coordination at $0 \mathrm{GPa}$ to more than fivefold coordination above $20 \mathrm{GPa}$, occurs as a consequence of pressure-induced IPT around $20 \mathrm{GPa}$. Moreover, our experimental and theoretical results show a strong elastic anisotropy of orpiment layers between 0 and $23 \mathrm{GPa}$, that confirms recent results [36] and open the door to use membranes of this $2 \mathrm{D}$ material in flexible and stretchable devices $[37,38]$. 
Additionally, our vibrational results show a closing of the phonon gap with increasing pressure up to $16 \mathrm{GPa}$ due to the large number of phonons with negative pressure coefficients (soft modes) at relatively low pressures. Our theoretical calculations have allowed us to provide a tentative symmetry assignment of the Raman-active modes in orpiment and explain their behavior under compression, which can help in identifying orpiment in ancient pigments in a non-destructive way [39].

Our electronic band structure calculations suggest that there is a progressive closing of the bandgap of $\alpha-\mathrm{As}_{2} \mathrm{~S}_{3}$ with increasing pressure leading from a trivial semiconductor to a trivial metal well above $60 \mathrm{GPa}$, which is in very good agreement with previous optical absorption measurements $[26,27,31]$ and suggest a possible phase transition above $42 \mathrm{GPa}$ due to the recently observed metallization of orpiment [33].

Finally, we show that all the changes observed in orpiment under compression, such as the strong decrease of the bandgap; the softening of many optical vibrational modes; the evolution of the electron localization function (ELF) along the As-S bonds; and the strong increase of the dielectric tensor components and Born effective charges, can be explained in the framework of the formation of metavalent bonding at HP, as recently proposed for other chalcogenides [40-45]. In summary, we show in the present paper that orpiment under compression is an example, together with GeSe [43], of a compound that develops a pressure-induced transition from covalent to metavalent bonding without the need of undergoing a firstorder structural phase transition. This feature contrasts to Se and Te, which undergo this change of bonding character after being subject of a first-order phase transition. Moreover, we propose that the formation of metavalent bonding may explain the behavior under compression of other group-15 chalcogenides, so our results pave the way for extending the study of the transformation from covalent to metavalent bonding to other group-15 chalcogenides and related compounds.

\section{B Results}

\section{B.1 Structural and vibrational characterization of $\alpha-A s_{2} S_{3}$ at room} conditions

As already commented, $\alpha-\mathrm{As}_{2} \mathrm{~S}_{3}$, with monoclinic $P 2_{1} / c$ space group at room conditions, is a layered material with corrugated (or zigzag) layers extended along the ac-plane that are linked by vdW interlayer interactions along the $b$-axis (Figure 1(a)). Each corrugated layer is formed by heart-shaped six-member rings of corner-sharing $\mathrm{AsS}_{3}$ pyramids (Figure 1(b)) Traditionally, the zigzag layers have been understood as formed by spiral AsS chains extended along the $a$-axis and held together by interchained $\mathrm{S}$ atoms, which allow the link between the chains along the $c$-axis (see Figure 1(c)). On the other hand, zigzag layers can also be seen as a W-shape formation of molecules (S1-As2-S3As1-S2) where each molecule is linked with four adjacent inverted $\mathrm{W}$-shape molecules, mostly along the $c$-axis due to covalent bonding (see Figure 1(d))
At room conditions, each As1 and As 2 atom is linked by covalent bonds to one S1, one S2 and one S3 atom at distances around $2.3 \AA$, leading to an As threefold-coordination (see pyramids in Figure 1(a)). Additionally, each As1 and As2 atom has two farther $\mathrm{S}$ neighbors on the same layer at distances above $3.0 \AA$ (see dashed lines inside the layers in Figures 1(a) and 1(b)) and other two $\mathrm{S}$ neighbors on an adjacent layer at distances above 3.5 $\AA$ (see dashed lines between the layers at the top part of Figures $\mathbf{1}(\mathrm{a})$ and $\mathbf{1}(\mathrm{d})$ ). In the following section, we will discuss the importance of these long As-S intra- and interlayer distances (above $3 \AA$ at room pressure) in orpiment at high pressure.
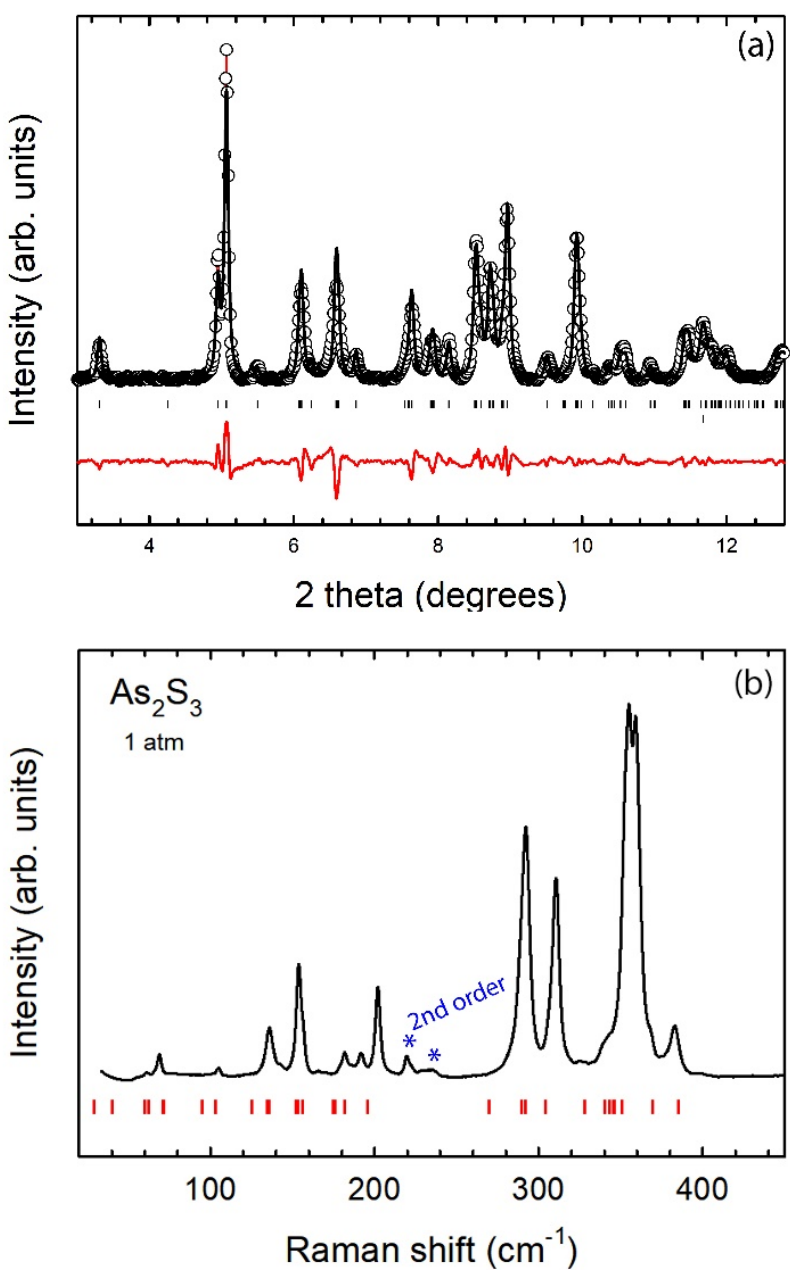

Figure 2. Structural and vibrational characterization of $\alpha-\mathrm{As}_{2} \mathrm{~S}_{3}$ at room conditions: (a) Powder XRD pattern (circles). Le Bail analysis (black solid line) and residuals (red solid line) are also plotted. (b) RS spectrum. Bottom marks indicate the theoretical Raman-active mode frequencies from calculations including vdW interactions. Asterisk indicates $2^{\text {nd }}$ order Raman features.

According to the difference of the As-S distances, we can consider orpiment as an arrangement of $\mathrm{AsS}_{3}$ pyramids forming $\mathrm{AsS}_{3} \mathrm{E}$ tetrahedral units, where $\mathrm{E}$ refers to the lone electron pair (LEP) of the As atoms. Therefore, each layer can be described alternatively by an arrangement of two tetrahedral $\mathrm{AsS}_{3} \mathrm{E}$ units; i.e. those formed by the two inequivalent As atoms, each surrounded by three $S$ atoms and the corresponding cation LEP that points towards the interlayer space. The strong As LEP 
Table 1 Experimental and theoretical (with vdW) lattice parameters corresponding to the $P 2_{1} / c$ phase of $\alpha-A s_{2} S_{3}$ at ambient conditions. Experimental and theoretical values from Refs. 10, 11, 33 and $\mathbf{7 2}$ are given for comparison. All data have been expressed in the standard $P 2_{1} / c$ setting for comparison.

\begin{tabular}{|l|l|l|l|l|l|l|}
\hline $\begin{array}{l}\text { Lattice } \\
\text { parameters }\end{array}$ & $\begin{array}{l}\text { Experimental } \\
\text { (This work) }\end{array}$ & $\begin{array}{l}\text { Theoretical } \\
\text { (This work) }\end{array}$ & $\begin{array}{l}\text { Experimental } \\
\text { (Ref. 11) }\end{array}$ & $\begin{array}{l}\text { Experimental } \\
\text { (Ref. 10) }\end{array}$ & $\begin{array}{l}\text { Theoretical } \\
\text { (Ref. 33) }\end{array}$ & $\begin{array}{l}\text { Theoretical } \\
\text { (Ref. 72) }\end{array}$ \\
\hline$a(\AA)$ & $4.2626(5)$ & 4.2608 & $4.256(2)$ & $4.22(5)$ & 4.22 & 4.22 \\
\hline$b(\AA)$ & $9.6056(7)$ & 9.6289 & $9.577(5)$ & $9.57(2)$ & 9.65 & 9.57 \\
\hline$c(\AA)$ & $12.1836(12)$ & 12.3084 & $12.191(5)$ & $12.18(4)$ & 12.27 & 12.1839 \\
\hline$\beta(0)$ & $110.054(7)$ & 109.84 & $109.75(8)$ & $109.8(5)$ & 109.59 & 109.85 \\
\hline$V_{0}\left(\AA^{3}\right)$ & $468.61(5)$ & 475.00 & 467.68 & 462.8 & 471.2 & 462.80 \\
\hline
\end{tabular}

distorts the electronic distribution and the geometry of the polyhedral units leading to the layered structure of orpiment. The layered structure of orpiment bears similarities with the chain-like structure of the valentinite mineral $\left(\beta-\mathrm{Sb}_{2} \mathrm{O}_{3}\right)$, where the W-shape molecules (linked through a helical form) lead to the formation of an acicular or quasimolecular crystal [46]. On the other hand, the layered structure of orpiment is isostructural to that of $\alpha-\mathrm{As}_{2} \mathrm{Se}_{3}$ [47] and belongs to the same space group that the claudetite mineral $\left(\mathrm{m}-\mathrm{As}_{2} \mathrm{O}_{3}\right)$; however, claudetite is not isostructural to orpiment, because the layered structure of the former is due to the arrangement of $\mathrm{AsO}_{3}$ molecules in a buckled structure [48] as in black phosphorous [49]. The layered structure of orpiment also bears some resemblance to the zigzag layered structure recently described for $\mathrm{Sb}_{2} \mathrm{~S}_{3}, \mathrm{Sb}_{2} \mathrm{Se}_{3}$, and $\mathrm{Bi}_{2} \mathrm{~S}_{3}$ [50-52], crystallizing in the orthorhombic Pnma structure, and to that of $\alpha-\mathrm{As}_{2} \mathrm{Te}_{3}$, crystallizing in the monoclinic $C 2 / m$ phase [53]. In these former group-15 $A_{2} X_{3}$ sesquichalcogenides, the layers are clearly composed by spiral chains held together through long and weak $A-X$ bonds, whereas in $\mathrm{As}_{2} \mathrm{~S}_{3}$ the chains are connected through short and strong $A-X$ bonds. Another difference found between these systems, is regarding the cation coordination in the different structures at room pressure. In $\alpha-\mathrm{As}_{2} \mathrm{~S}_{3}$, the two inequivalent $A$ s atoms are threefold-coordinated; in $\mathrm{Sb}_{2} \mathrm{~S}_{3}, \mathrm{Bi}_{2} \mathrm{~S}_{3}$ and $\mathrm{Sb}_{2} \mathrm{Se}_{3}$, there is an average fourfold coordination because one cation has threefold coordination and the other has fivefold coordination; and in $\alpha-\mathrm{As}_{2} \mathrm{Te}_{3}$, As atoms have a coordination between five and six. This means that the larger cation or the larger anion favors an increase of cation coordination probably due to the smaller cation LEP effect [54].

The experimental XRD pattern of our sample at room conditions (see Figure 2(a)) shows that the sample corresponds to the $\alpha$-phase without the presence of additional phases or impurities, within the detection limits of the technique. The pattern was fitted to a monoclinic structure ( $P 2_{1} / c$ space group), with small residuals and a correlation factor $R_{w p}$ of $10 \%$, yielding the following lattice parameters: $a=4.2626(5) \AA, b=9.6056(7)$

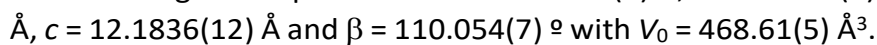

In Table 1 we can observe that these values are in good agreement with previously reported experimental values $[10,11,13]$, with our theoretical calculated lattice parameters and with other previously published theoretical data $[33,55]$. The theoretical atomic parameters of the five inequivalent atomic sites (three $\mathrm{S}$ and two $\mathrm{As}$ atoms) of $\alpha-\mathrm{As}_{2} \mathrm{~S}_{3}$ are also given in Table S1 in the Supporting Information (SI) section for comparison with experimental and theoretical values.

As regards to the vibrational properties of $\alpha-A s_{2} S_{3}$, group theory predicts sixty vibrational modes at the (Brillouin BZ center with the following mechanical representation [56]:

$\Gamma_{60}=15 A_{g}(R)+13 B_{u}(I R)+15 B_{g}(R)+14 A_{u}(I R)+1 A_{u}+2 B_{u}$

where $\mathrm{g}$ (gerade) modes are Raman-active $(\mathrm{R})$ and $\mathrm{u}$ (ungerade) modes are infrared-active (IR), except one $A_{u}$ and two $B_{u}$ modes, which are acoustic modes. Therefore, $\alpha-\mathrm{As}_{2} \mathrm{~S}_{3}$ has 30 Ramanactive modes and 27 IR-active modes.

Figure 2(b) shows the unpolarized RS spectrum of orpiment at room conditions together with the theoretically-predicted Raman-active mode frequencies at room pressure. The values of the experimental and theoretical frequencies of the Ramanactive modes of $\alpha-\mathrm{As}_{2} \mathrm{~S}_{3}$ at room pressure are summarized in Table 2. The RS spectrum of orpiment at room pressure clearly shows 19 out of the 30 theoretically-predicted Raman-active modes and a quite good agreement between our experimental and theoretical frequencies (including vdW interactions). The spectrum is similar to those previously reported, with a doublet between 60 and $70 \mathrm{~cm}^{-1}$, a phonon gap between 200 and 290 $\mathrm{cm}^{-1}$ and a triplet around $360 \mathrm{~cm}^{-1}[\mathbf{1 4 - 2 9 , 3 3 ]}$. Unfortunately, we have not been able to observe modes below $40 \mathrm{~cm}^{-1}$ due to the limit imposed by the edge filter of our spectrometer.

The vibrational spectrum of orpiment at room pressure is separated between a low-frequency region (below $200 \mathrm{~cm}^{-1}$ ) and a high-frequency region (above $260 \mathrm{~cm}^{-1}$ ) with a phonon gap between these regions. Some Raman features are observed inside the phonon gap of $\alpha-A s_{2} S_{3}$, which are assumed to be second-order modes, so they will not be further commented in 
this work. Among the measured RS modes below $250 \mathrm{~cm}^{-1}$ and above $280 \mathrm{~cm}^{-1}$, the more intense modes are those of the highfrequency region between 350 and $360 \mathrm{~cm}^{-1}$. Due to the large number of Raman-active modes located within a small frequency region of the RS spectrum at room pressure and the broadening of experimental peaks, it is almost impossible to identify the modes by solely using the RS spectrum at room pressure and its comparison with lattice-dynamics $a b$ initio calculations. Therefore, a tentative assignment of experimental peaks will be performed in a forthcoming section taking into account the HP dependence of the experimental and theoretical Raman-active mode frequencies.

The atomic vibrations of several characteristic Raman- and IRactive modes of $\alpha-A s_{2} S_{3}$ have been visualized with the aid of the Jmol Interface for Crystallographic and Electronic Properties (JICE) interface [57] (see Figures S1 to S14 in the SI) and discussed in the SI. An interesting feature is that many vibrations evidence that atoms of the same chain (As1, S1, As2, and S2) vibrate inphase or out-of-phase, whereas S3 atoms interconnecting the chains vibrate in a quite different fashion than the other four inequivalent atoms. This observation clearly evidences the chain-like nature of the layers of orpiment in good agreement with a previous vibrational study [24].

As a layered material, some of the most important vibrational modes of $\alpha-\mathrm{As}_{2} \mathrm{~S}_{3}$ are the rigid layer modes. Our calculations confirm that the two lowest-frequency modes located at 27 and $38 \mathrm{~cm}^{-1}$, and attributed to the $\mathrm{Ag}_{\mathrm{g}}{ }^{1}$ and $\mathrm{Ag}^{2}$ modes, correspond to the rigid shear layer modes of orpiment (Figure S1 in the SI). Moreover, our calculations show that the compressional or longitudinal rigid layer mode is the $\mathrm{Bg}^{1}$ mode theoretically predicted at $62 \mathrm{~cm}^{-1}$ (Figure $\mathbf{S 2}$ in the $\mathrm{SI}$ ). This frequency value is in good agreement with previous estimations of the compressional mode [22]. At room pressure, we have observed this mode is within a region where it is overlapped with interchain vibrational modes (see comments in the $\mathrm{SI}$ ), as previously suggested [24].

As regards other vibrational modes of orpiment, those of the high-frequency region mainly correspond to As-S stretching modes located between 350 and $400 \mathrm{~cm}^{-1}$ and to a mixture of As-S stretching and bending modes between 260 and $350 \mathrm{~cm}^{-1}$. On the other hand, modes located below $200 \mathrm{~cm}^{-1}$ correspond to pure As-S bending modes, being those below $100 \mathrm{~cm}^{-1}$ mainly related to rotations and translations of the spiral chains, which can be seen as rigid units. This distribution of modes agrees well with previous works, that assigned the As-S bending modes and As-S stretching modes near 200 and $400 \mathrm{~cm}^{-1}$, respectively $[18,58]$. In fact, the modes close to 380 and $350 \mathrm{~cm}^{-1}$ can be assigned to the antisymmetric and symmetric As-S stretching vibrations inside the spiral chains, respectively, in good agreement with previous estimations [28].

\section{B.2 Structural characterization of $\alpha-\mathrm{As}_{2} \mathrm{~S}_{3}$ at HP}

Figure 3 shows powder HP-XRD patterns of orpiment at selected pressures up to $26.6 \mathrm{GPa}$. XRD patterns were analyzed by means of Le Bail fits to the monoclinic $P 2_{1} / C$ structure, which is stable up to the maximum achievable pressure. Le Bail analysis of this material allowed the possibility of obtaining the structural parameters of $\alpha-A s_{2} S_{3}$ at different pressures (see Figure 4). A progressive increase of the Bragg peaks width was observed and explained in the framework of a progressive loss of the hydrostatic conditions. The layered nature of this material avoid obtaining fine powder for this measurement, which led to the lack of Rietveld refinement of our XRD patterns. A monotonous decrease of the unit-cell volume of orpiment up to $26.6 \mathrm{GPa}$ is observed in Figure $\mathbf{4}(\mathrm{a})$, where experimental volume data at several pressures have been compared with DFT calculations, both including and without including $\mathrm{vdW}$ interactions. At low pressures, the pressure dependence of the experimental volume agrees quite well with the theoretical simulation when vdW interactions are included. However, experimental data above $10 \mathrm{GPa}$ show a smaller agreement with these calculations and much better agreement with calculations that do not include vdW interactions. The above experimental result indicates that orpiment is more incompressible above $10 \mathrm{GPa}$, likely due to the loss of quasihydrostatic conditions of the pressure-transmitting medium above this pressure.

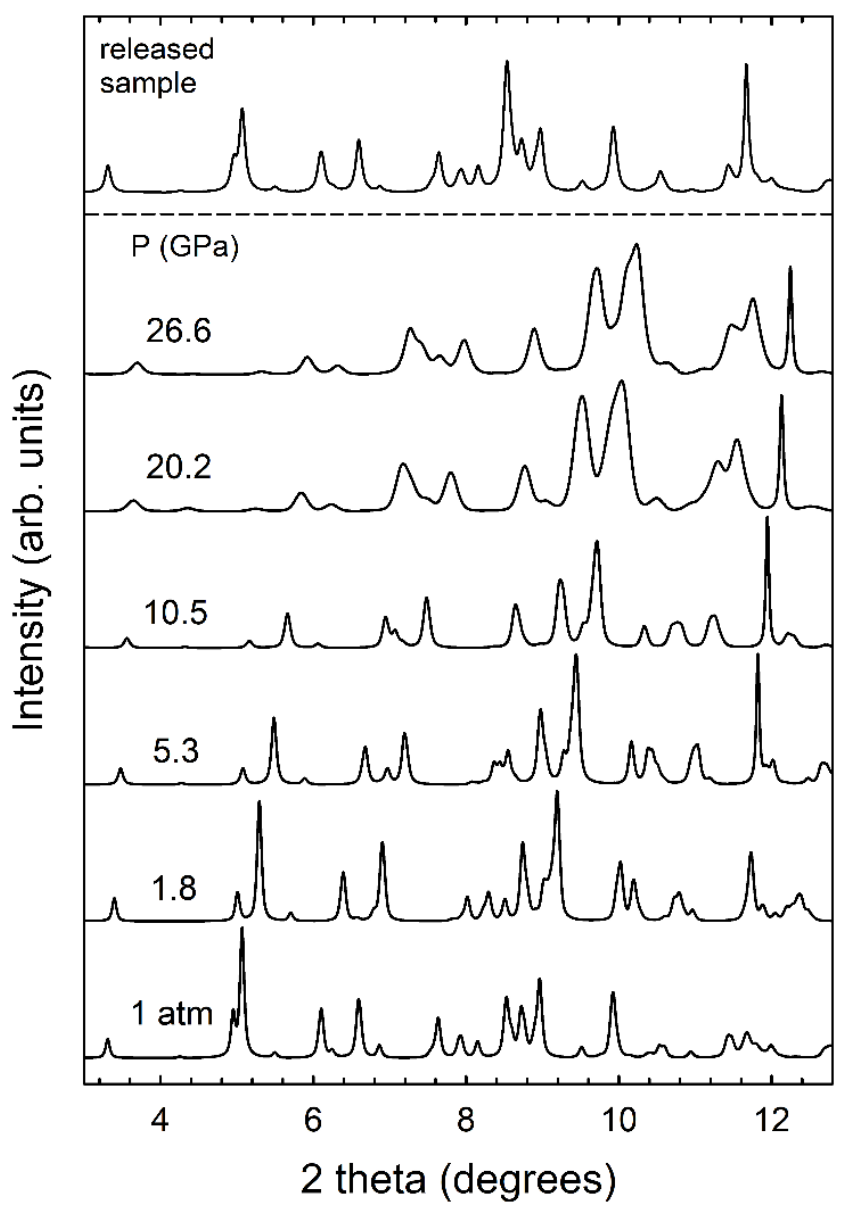

Figure 3. Powder HP-XRD patterns of $\alpha-\mathrm{As}_{2} \mathrm{~S}_{3}$ at selected pressures up to 26.6 GPa. Patterns are shifted vertically for comparison.

The fit of pressure vs. experimental volume up to $10 \mathrm{GPa}$ through a third-order Birch-Murnaghan Equation of State (BMEoS) [59], according to the trend showed by its $F-f$ plot (Figure 
S15 in SI) yields a zero-pressure volume, bulk modulus and pressure derivative of the bulk modulus of: $V_{0}=468.61(5) \AA^{3}$, $B_{0}=13.3(5) \mathrm{GPa}$ and $B_{0}{ }^{\prime}=8.9(5)$, respectively. The volume at zero pressure, $V_{0}$, was fixed to the value measured outside the DAC and the order of the BM-EoS was determined by the monotonously positive slope of the Eulerian strain vs normalized pressure plot of the theoretically simulated data. This plot agrees with highly compressible non-covalent compounds with large cation LEP activity, as it has been observed for structurally-related sesquichalcogenides, such as $\mathrm{Sb}_{2} \mathrm{~S}_{3}, \mathrm{Sb}_{2} \mathrm{Se}_{3}, \mathrm{Bi}_{2} \mathrm{~S}_{3}$ and $\alpha-\mathrm{As}_{2} \mathrm{Te}_{3}$ and characterized by bulk modulus pressure derivatives larger than $4[52,53]$.

Our experimental bulk modulus and pressure derivative under hydrostatic conditions for orpiment can be compared to our theoretical data with and without vdW interactions. As expected in the description of Figure 4(a), the theoretical bulk modulus is closer to the experimental one when calculations include $\mathrm{vdW}$ interactions $\left(B_{0}=12.7(5) \mathrm{GPa}\right)$ than when calculations do not include them ( $\left.B_{0}=16.1(12) \mathrm{GPa}\right)$. Nevertheless, the theoretical pressure derivative of the bulk modulus at zero pressure is considerably smaller than the experimental value $\left(B_{0}{ }^{\prime}=8.9(5)\right)$ in the case of simulations with $\mathrm{vdW}$ interactions $\left(B_{0}{ }^{\prime}=7.0(14)\right)$ than in simulations without $\mathrm{vdW}$ interactions $\left(B_{0}^{\prime}=7.9(6)\right)$. A comparison of these results with similar compounds, such as the claudetite polymorph of $\mathrm{As}_{2} \mathrm{O}_{3}$ or $\alpha-\mathrm{As}_{2} \mathrm{Te}_{3}$, indicates that orpiment presents a similar value of the bulk modulus to the former $\left(B_{0}=15.5(4) \mathrm{GPa}\right)$ [60], but a much smaller value to the bulk modulus than the latter $\left(B_{0}=\right.$ 24(3) GPa) [53]. This result is consistent with the higher LEP activity of As atom in oxides and sulfides than in selenides and tellurides [54].
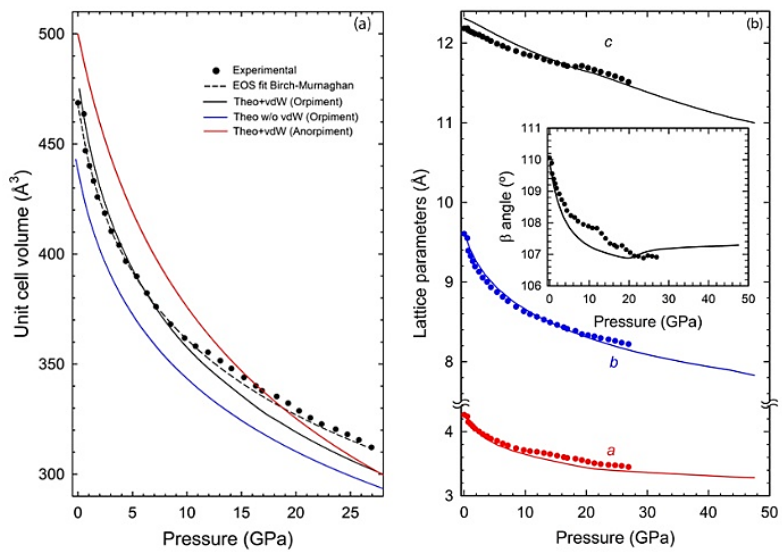

Figure 4. (a) Experimental (symbols) and theoretical (solid lines) pressure dependence of the unit-cell volume in $\alpha-\mathrm{As}_{2} \mathrm{~S}_{3}$. Black (blue) solid lines represent data from calculations including (do not including) vdW interactions. Dashed lines correspond to experimental data fit to a $3^{\text {rd }}$ order BM-EoS. (b) Experimental (symbols) and theoretical (solid lines) pressure dependence of the lattice parameters and monoclinic $\beta$ angle (inset) in $\alpha-\mathrm{As}_{2} \mathrm{~S}_{3}$. All calculations include vdW interactions.

The pressure dependence of the experimental and theoretical lattice parameters of $\alpha-\mathrm{As}_{2} \mathrm{~S}_{3}$ up to $26.6 \mathrm{GPa}$ also shows a monotonous and smooth decrease (see Figure 4(b)). The good agreement of the theoretical behavior of volume and lattice parameters with our experimental data at room temperature allows us to conclude that no first-order phase transition occurs throughout the whole range of studied pressures. Furthermore, the pressure dependence of our lattice parameters do not evidence anomalies at the region close to $10 \mathrm{GPa}$, which could be indicative of any structural transition as already suggested [30]. Therefore, our results are in good agreement with recently published theoretical data [33].

The analysis of the axial compressibility in monoclinic structures requires a more complex analysis than for more symmetric phases because the $\beta$-angle is not 90 , so the directions of maximum and minimum compressibility of the compound are usually not along any of the three crystallographic axes. Therefore, we have analyzed the compressibility of the material by calculating and diagonalizing the experimental and theoretical isothermal compressibility tensor $\beta_{\mathrm{ij}}$ at different pressures (details are given in the $\mathrm{SI}$ ). This tensor is a symmetric second rank tensor that relates the state of strain of a crystal to the pressure change that induced it [61] and it has been obtained with the finite Eulerian approximation as implemented in the Win_Strain package [62]

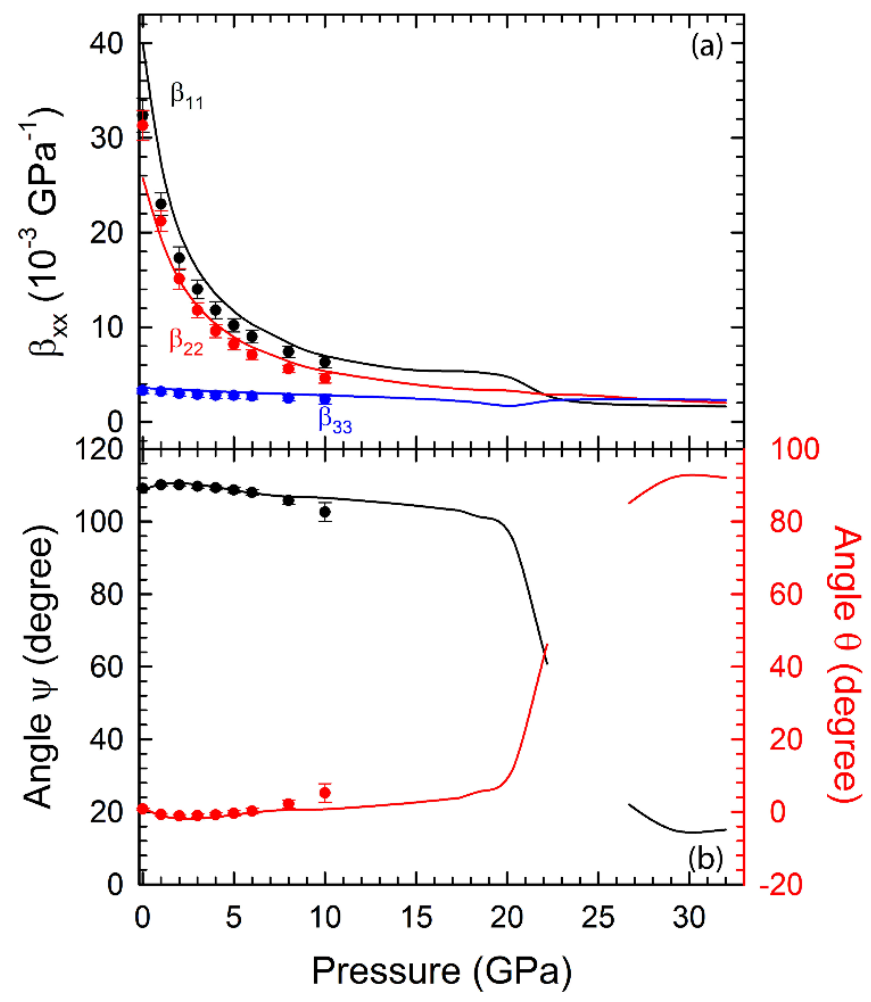

Figure 5. (a) $\beta_{x x}$ coefficients of the compressibility tensor that indicates the compressibility along the different crystallographic axes. (b) Angle of maximum compressibility $\psi$ relative to the $c$-axis (from $c$ to $a$ ) or equivalently $\theta$ relative to the $a$-axis (from $a$ to $c$ ). Solid lines represent data from calculations and symbols data from our experiments.

Figures $\mathbf{5}(\mathbf{a})$ and $\mathbf{5}(\mathbf{b})$ describe the pressure dependence of the tensor elements (numerical data are provided in Tables S1 and S2 in the SI) corresponding to the compressibilities along the different axis and the direction of maximum compressibility with respect to the $c$-axis $(\psi)$ or to the $a$-axis $(\theta)$, respectively. 

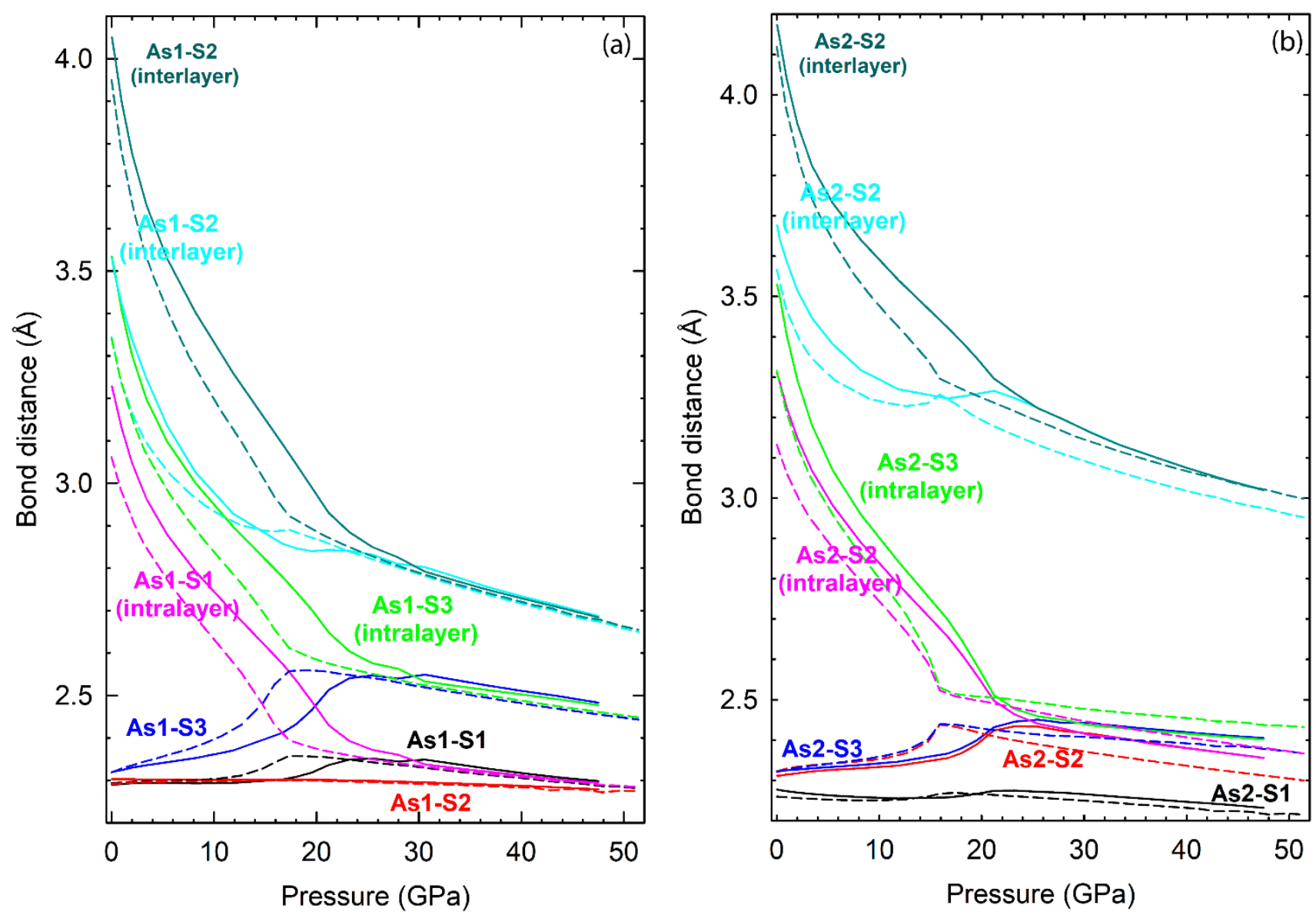

Figure 6. Evolution under pressure of the theoretical As-S interatomic distances of $\alpha-A s_{2} S_{3}$. (a) As1 and its next-neighbor $S$ atoms. (b) As2 and its nextneighbor $\mathrm{S}$ atoms. Dashed (solid) lines represent data from theoretical calculations that include (do not include) vdW interactions.

According to these figures, the $a$-axis (the spiral axis) is more compressible than the $b$-axis (the axis perpendicular to the layers) up to $23 \mathrm{GPa}$. This is a surprising result for layered materials, where the axis perpendicular to the layers is usually the most compressible one; however, it is coherent with the spiral chain-like nature of the layers in orpiment and the small connectivity of the spiral layers along the $a$-axis. Moreover, the compressibility of the $a$-axis $\left(\beta_{11}\right)$ is much larger than that of the $c$-axis $\left(\beta_{33}\right)$. This high intralayer elastic/mechanic anisotropy is consistent with that recently found in $\mathrm{As}_{2} \mathrm{~S}_{3}$ nanolayers, which is similar to that of black phosphorous [36] and certifies that orpiment layers can be potentially used in flexible, stretchable, orientation-dependent and frequency tunable electronic, optoelectronic and optomechanic devices $[37,38]$.

The compressibility trend among the different crystallographic directions remains constant $\left(\beta_{11}>\beta_{22}>\beta_{33}\right)$ up to $23 \mathrm{GPa}$, being all axial compressibilities positive, thus indicating a monotonous compression of the structure along the three main crystallographic axes up to $23 \mathrm{GPa}$. This result is somewhat in disagreement with previous estimates by Besson et al. [26], who found positive and negative values for the compressibilities along the $a$ - and $c$-axis of the layer plane, respectively, and suggested a compression of the layers in the direction perpendicular to the spiral chains and an expansion of the spiral chains along the helicoidal axis, similar to what occurs in trigonal Se and Te $[63,64]$

The reliability of our theoretical calculations allowed us to obtain the theoretical compressibility tensor up to $32 \mathrm{GPa}$. A sudden regularization of the three axial compressibilities to roughly the same value is attained around $23 \mathrm{GPa}$, with a quick change of the direction of maximum compressibility around this pressure. At $24 \mathrm{GPa}$, the direction of maximum compressibility is found to be along the $b$-axis ([010] direction); reason why we do not display any value on Figure 5. However, above $27 \mathrm{GPa}$, the direction of maximum compressibility is again found to be within the ac-plane, but now it is close to the $c$-axis. In particular, $\Psi=15(4) \circ$ and $\theta=92(4) \circ$ at $32 \mathrm{GPa}$. The drastic change of the direction of the maximum compressibility observed above $23 \mathrm{GPa}$ can be considered as a more significant feature of the variation of properties associated to the lowpressure structure.

For completeness, the experimental and theoretical pressure dependence of the axial ratios in $\alpha-\mathrm{As}_{2} \mathrm{~S}_{3}$ is shown in Figure S16 in the SI. The monotonous trend of all the axial ratios is well reproduced by our theoretical calculations including vdW interactions. A closer look into the slopes reveals a clear change of tendency above $20 \mathrm{GPa}$, where all three ratios seem to become insensitive to pressure up to $50 \mathrm{GPa}$. A similar behavior is observed in the pressure dependence of the $\beta$-angle (inset of 
Figure $\mathbf{4}(\mathbf{b})$ ). In some previous works, the change in the axial ratios of several group-15 sesquichalcogenides has been considered as a proof for the occurrence of a pressure-induced electronic topological transition (ETT) $[\mathbf{5 0 , 6 5 , 6 6 ]}$, since a minimum of the $c / a$ ratio has previously been observed to be coincident with the occurrence of a pressure-induced ETT in $\alpha$ $\mathrm{Bi}_{2} \mathrm{Se}_{3}, \alpha-\mathrm{Sb}_{2} \mathrm{Te}_{3}$, and $\alpha-\mathrm{Bi}_{2} \mathrm{Te}_{3}$ [67]. However, in recent works $[52,68]$, this fact has been put into question because the minimum of the $c / a$ ratio can be simply originated by a change of the ratio of interlayer/intralayer forces. Therefore, the change of the minimum of the $c / a$ ratio does not necessarily warrant a change in the electronic density of states near the Fermi level leading to an ETT; however, it can be indicative of an IPT, as recently suggested for orpiment [33].

To prove that the change of the axial ratios is indicative of a pressure-induced IPT, we have analyzed the pressure dependence of the internal atomic parameters of the five inequivalent atomic sites in $\alpha-\mathrm{As}_{2} \mathrm{~S}_{3}$ (see Figure $\mathbf{S 1 7}$ in the $\mathrm{SI}$ ). The most noticeable results are those observed for the three coordinates of the two inequivalent As atoms, which show a clear trend towards certain fixed coordinates above 18 (25) $\mathrm{GPa}$, as obtained for calculations without (with) vdW interactions. This result occurs at similar pressures where the change of the direction of the maximum compressibility was observed, which clearly indicates a modification of the HP behavior of the low-pressure phase.

A remarkable result, derived from the pressure dependence of the atomic coordinates, is the grouping and regularization of many theoretical As-S interatomic distances around 18 (25) GPa, without (with) vdW interactions (see Figure 6). As a consequence, there is an increase of As1 coordination from threefold at $0 \mathrm{GPa}$ to more than fivefold $(5+2)$ above $20 \mathrm{GPa}$ (see Figure 1e) because, on one hand, all As1-S1 and As1-S3 intralayer distances become similar, thus giving a fivefold coordination and, on the other hand, two additional As1-S2 interlayer distances become smaller than $3 \AA$. Similarly, there is an increase of As2 coordination from threefold at $0 \mathrm{GPa}$ to fivefold above $20 \mathrm{GPa}$ that stems from the equalization of the As2-S2 and As2-S3 intralayer distances. In summary, we can conclude that above $20 \mathrm{GPa}$ the As1 (As2) polyhedral units of orpiment pass from a threefold coordination towards a sevenfold (fivefold) coordination without changing the space group.

A more detailed analysis of the As polyhedral units (see Figures S18 to S20 in the SI) shows how both As1 and As2 polyhedral units remain in a threefold effective coordination below $10 \mathrm{GPa}$. However, above this critical pressure, there is a progressive increase of the effective coordination up to $22 \mathrm{GPa}$, where the As2 polyhedron remains in fivefold effective coordination, whereas that of the As1 polyhedral unit increases up to sixfold effective coordination (Figure $\mathbf{S 2 0}$ in the $\mathrm{SI}$ ). We must note that in distorted polyhedral units (values very different from 0 in Figure S19 in the SI), the effective coordination number does not describe properly the coordination of the polyhedral unit because some of the interatomic distances are longer than others, thus leading to an effective coordination dominated by the next neighbors (Figure $\mathbf{S 2 0}$ in the $\mathrm{SI}$ ) but with large polyhedral volumes (Figure S18 in the SI). Above 20 GPa, the effective coordination of As1 is slightly larger than five despite there is a higher real coordination of $(5+2)$ due to the strong distortion of the polyhedral unit around As1, whereas for As2 the effective coordination (five) coincides with the real coordination due to the proximity of the interatomic distances. The above results between 0 and $20 \mathrm{GPa}$ are consistent with: i) the regularization of the axial compressibilities, ii) the trend towards fixed As coordinates found, and iii) the similar $x$ and $z$ coordinates showed by the Wyckoff sites of As1, S2, and S3 atoms, above 18 (25) GPa in our calculations without (with) vdW interactions. All these results indicate the presence of an IPT above $20 \mathrm{GPa}$, in agreement with a recent paper [33] and also with HP-XAS results that reported a change in the As environment above $10 \mathrm{GPa}$ and an increase of As coordination above $30 \mathrm{GPa}$ [30].

In summary, HP-XRD measurements and DFT calculations carried out up to $32 \mathrm{GPa}$ show that the effect of pressure upon the structure of $\alpha-A s_{2} S_{3}$ does not result in a first-order phase transition, but triggers a change of the trend of atomic coordinates, of axial ratios, and an increase of coordination of As atoms above $20 \mathrm{GPa}$; as well as a drastic change of the direction of the maximum compressibility above $23 \mathrm{GPa}$. All these features are in good agreement with published data $[30,33]$ and could be attributed to a pressure-induced IPT in orpiment above $20 \mathrm{GPa}$.

\section{B.3 Vibrational characterization of $\alpha-\mathrm{As}_{2} \mathrm{~S}_{3}$ at HP}

In order to study the effect of pressure on the vibrational properties of orpiment and better understand the HP behavior of $\alpha-A s_{2} S_{3}$, we have carried out HP-RS measurements on orpiment samples up to $14.5 \mathrm{GPa}$. Experimental results are compared to lattice-dynamics calculations of $\alpha-\mathrm{As}_{2} \mathrm{~S}_{3}$. On Figure 7 we show the room-temperature RS spectra of orpiment at selected pressures under hydrostatic conditions. In this figure, it is clear to observe the absence of any first-order PT in orpiment up to $14.5 \mathrm{GPa}$, in good agreement with our HP-XRD measurements and previously published results [30,33]. However, RS spectra show a much larger number of Raman modes between 2.0 and $4.1 \mathrm{GPa}$, despite the disappearance of the Raman modes in the phonon gap between 210 and 250 $\mathrm{cm}^{-1}$. As already commented, these modes in the phonon gap, together with a mode observed above $1 \mathrm{GPa}$ near $120 \mathrm{~cm}^{-1}$, are assumed to be second-order modes and will not be further discussed. No major changes of the RS spectra are observed between 4.1 and $14.5 \mathrm{GPa}$. In this context, it must be noted that the bandgap of orpiment ( $2.7 \mathrm{eV}$ at room pressure) decreases under pressure at a rate of $-0.14 \mathrm{eV} / \mathrm{GPa}[\mathbf{2 6 , 3 1}]$. This means that the bandgap equals the HeNe laser energy (1.96 eV) at 5.3 $\mathrm{GPa}$, so some resonance effects could be observed above ca. 4 GPa. However, no damage in the sample or redshift of frequency modes after long exposure time to the laser, which indicates the lack of radiation damage in our samples.

As already mentioned, it is very difficult to assign the features that show up in the room-pressure RS spectrum of $\alpha-A s_{2} S_{3}$. However, it is possible to perform a tentative peak assignment 
by studying the pressure dependence of the Raman-active modes in combination with lattice-dynamics calculations on the basis of the correlation of the frequencies and their pressure coefficients (see Table 2 and Figure 8(a)). Our observed frequencies and pressure coefficients are in good agreement with previous HP-RS studies $[\mathbf{2 3}, \mathbf{2 6}, \mathbf{2 7}, \mathbf{3 3}]$. Notably, the shear or transverse rigid modes $A_{g}{ }^{1}$ and $A_{g}{ }^{2}$ at 26 and $37 \mathrm{~cm}^{-1}$ and the first $B_{g}$ mode $\left(B_{g}^{2}\right.$ mode $)$ at $69 \mathrm{~cm}^{-1}$ show the largest relative increase of frequency under compression $(0.24,0.13$ and 0.15 $\mathrm{GPa}^{-1}$, respectively). Moreover, the $\mathrm{Bg}^{2}$ mode shows the experimental and theoretical largest pressure coefficient and mode Grüneisen parameter of all Raman-active modes (around $10.5 \mathrm{~cm}^{-1} / \mathrm{GPa}$ and 1.97 , respectively).

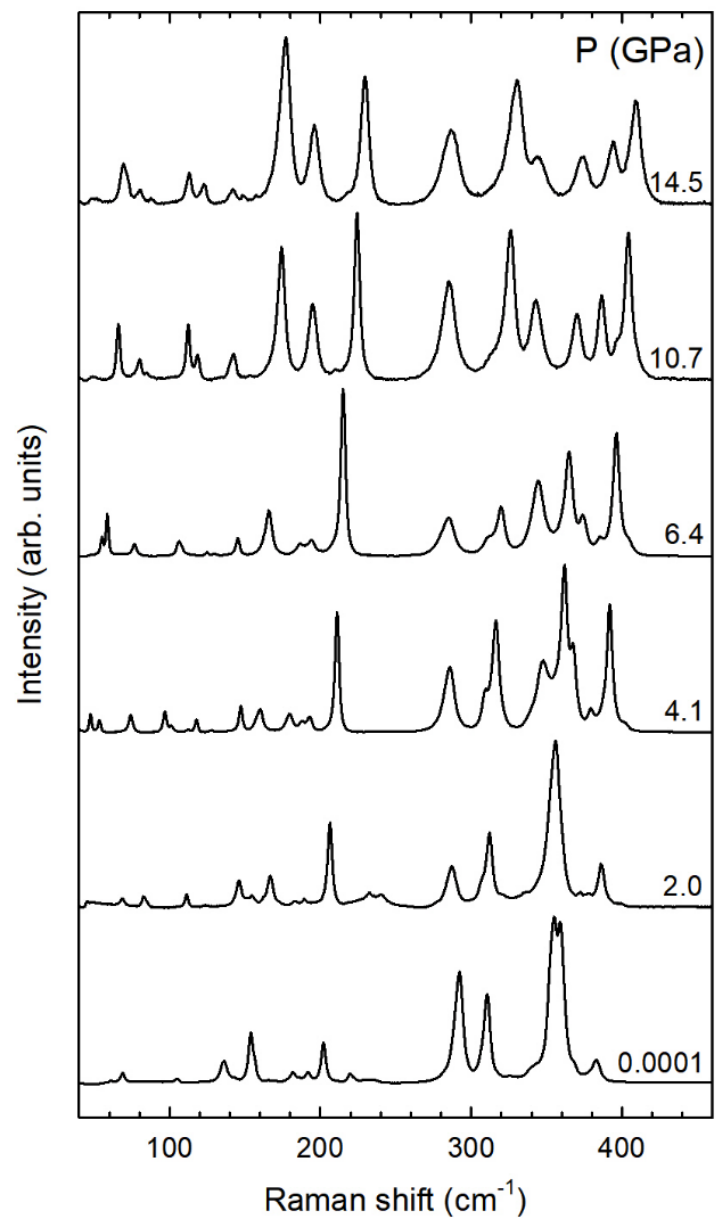

Figure 7. Room-temperature RS spectra of orpiment at selected pressures up to $15 \mathrm{GPa}$.

While the above results regarding the shear rigid layer $A_{g}$ modes are rather common in layer materials, the result regarding the $\mathrm{Bg}^{2}$ mode is quite surprising because the largest pressure coefficient is indeed expected for the compressional or longitudinal layer mode $\mathrm{Bg}^{1}$ located at $62 \mathrm{~cm}^{-1}$ (see Figure $\mathbf{S 2}$ in the $\mathrm{SI}$ ). It is well known that the compressional layer mode usually shows higher frequencies and pressure coefficients than those of the shear rigid layer modes in layered materials with $\mathrm{vdW}$ interactions between the layers. This common trend is due to the extraordinary increase of the stretching force constant between neighboring layers caused by the strong decrease of the interlayer distance, as discussed in a recent paper [69]. However, we have found that the $\mathrm{Bg}^{1}$ mode in orpiment (not observed experimentally) shows a rather small theoretical pressure coefficient (see Table 2). This anomalous pressure dependence of the compressional mode of orpiment can be understood by considering a frequency anticrossing (occurring already at room pressure) between the $\mathrm{Bg}_{\mathrm{g}}{ }^{1}$ and $\mathrm{Bg}^{2}$ modes. In fact, the large pressure coefficient of the "bare" $B_{g}{ }^{1}$ mode (see dashed line in Figure $\mathbf{8}(\mathbf{b})$ ) is so large, when compared to other Raman modes, that this mode undergoes anticrossings with up to four $\mathrm{B}_{\mathrm{g}}$ modes (up to $\mathrm{B}_{\mathrm{g}}{ }^{5}$ ) in the pressure range between 0 and $15 \mathrm{GPa}$. This anticrossing allows us to explain the small pressure coefficient of the $\mathrm{Bg}^{1}$ mode and the large pressure coefficient of the $\mathrm{Bg}_{\mathrm{g}}{ }^{2}$ mode of orpiment. Moreover, this puzzling behavior may explain why the compressional mode was not previously assigned, despite the frequency of the compressional mode was well identified in a previous work [22] as well as the pressure coefficient of the $\mathrm{Bg}^{2}$ mode [23].

As observed in Figure $\mathbf{8}(\mathbf{a})$, experimental and theoretical frequencies of Raman-active modes do not show a simple monotonic behavior with increasing pressure. In fact, many Raman modes exhibit a complex behavior under pressure with crossings and anticrossings of modes with different and equal symmetries, respectively. This complex behavior stems from the presence of 30 Raman-active modes in a small frequency region between 30 and $400 \mathrm{~cm}^{-1}$, similarly to what has been found in monoclinic $\alpha-\mathrm{As}_{2} \mathrm{Te}_{3}$ [53]. Despite the complex behavior observed in our theoretical Raman-active modes, several experimental modes have shown a behavior consistent with the theoretical modes. In particular, we have found an experimental reduction of the phonon gap with pressure, an anticrossing of Raman modes close to $150 \mathrm{~cm}^{-1}$ around $2 \mathrm{GPa}$, and the splitting of several Raman modes located near $350 \mathrm{~cm}$ 1 , which are in good agreement with previous results $[26,27,33]$. Such behaviors are supported by our lattice-dynamics calculations for the pressure dependence of the $\mathrm{Ag}_{\mathrm{g}}{ }^{9}$ and $\mathrm{Ag}^{10}$ modes, the $A_{g}{ }^{5}$ and $A_{g}{ }^{6}$ modes and the $A_{g}{ }^{12}, A_{g}{ }^{13}$ and $A_{g}{ }^{14}$ modes, respectively (see Figure $\mathbf{8}(\mathbf{a})$ ).

A striking feature of orpiment is the large number of Raman modes with negative pressure coefficients at room pressure. This is confirmed by both experimental and theoretical data. In particular, modes located at 155, 291, 325, 356 and $400 \mathrm{~cm}^{-1}$ show softening under pressure in rather good agreement with previous measurements [23,33]. As noted by Besson et al. [26], negative pressure coefficients of internal modes have been observed in Raman measurements of other chain-like structures, such as trigonal S, Se and Te [70-72], but never for ring molecules, like those found in orthorhombic $S$ [23]. In trigonal $\mathrm{S}$, Se and Te, the soft Raman phonon is the $\mathrm{A}_{1}$ mode (the breathing mode of the chains at the $a b$-plane [73]) and it can be related to the expansion of the $c$-axis, caused by the increase of the Se-Se intra-chain distance, at the expense of the contraction of the $a$-axis, caused by the decrease of the Se-Se inter-chain distance. In orpiment, the situation is quite different because all three $a-, b$-, and $c$-axes suffer a contraction under compression so the explanation for the negative pressure coefficients is not related to the elongation of any axis. 

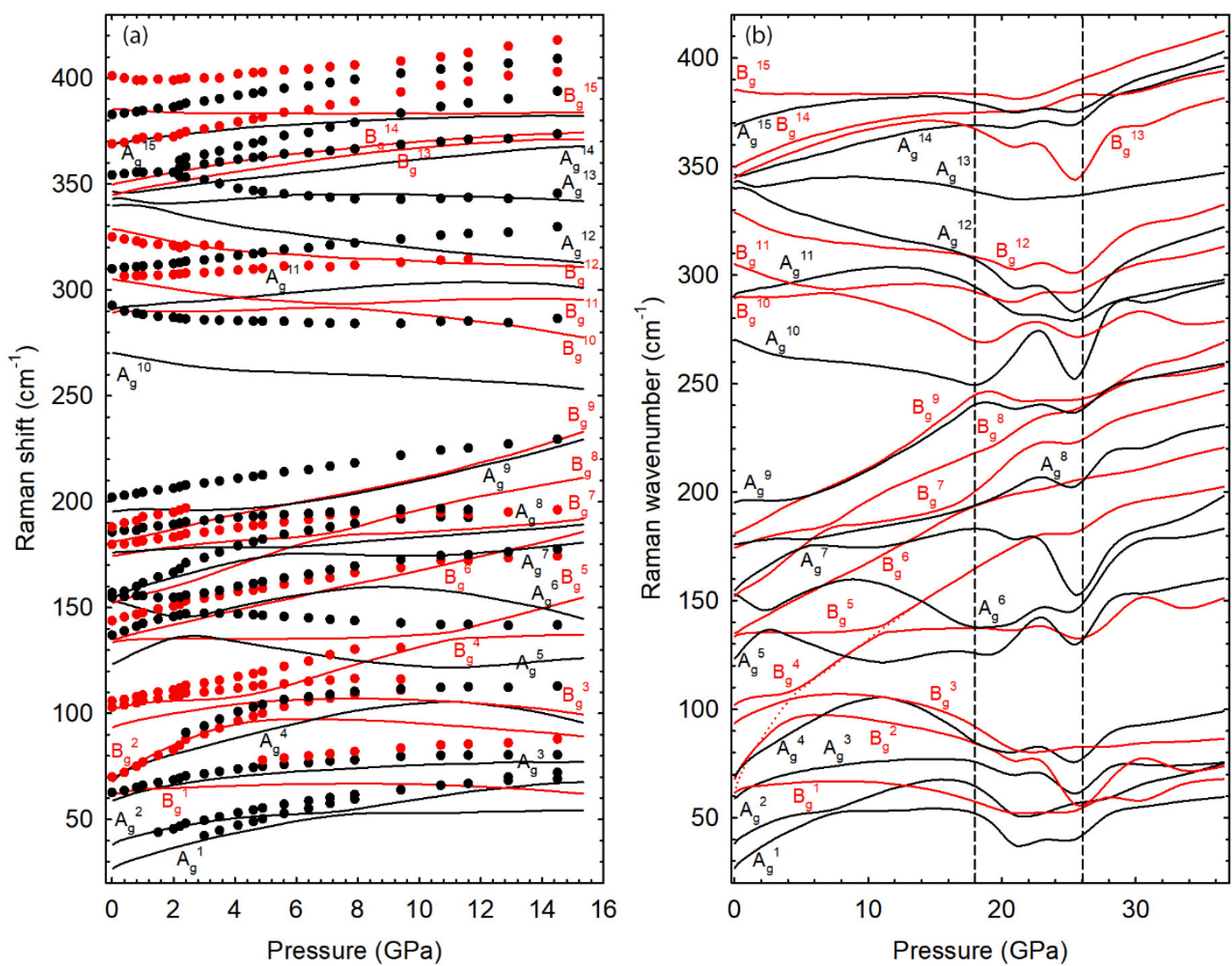

Figure 8. (a) Experimental (symbols) and theoretical (lines) pressure dependence of the Raman-active mode frequencies of $\mathrm{As}_{2} \mathrm{~S}_{3}$ up to $16 \mathrm{GPa}$. Black (red) color represents $A_{g}\left(B_{g}\right)$ Raman-active modes. Theoretical curves correspond to calculations with vdW interaction. (b) Pressure dependence of the theoretical Raman-active mode frequencies of $\mathrm{As}_{2} \mathrm{~S}_{3}$ up to $36 \mathrm{GPa}$. Red dotted line represents the tentative pressure dependence of the "bare" $\mathrm{B}_{\mathrm{g}}{ }^{1}$ mode (the compressional rigid layer mode) if no anticrossing would occur with other modes of the same symmetry. Note that a change in the pressure coefficient of the "bare" $\mathrm{Bg}^{1}$ mode observed near $4 \mathrm{GPa}$ and that a considerable softening of some vibrational modes is observed between 18 and $26 \mathrm{GPa}$.

Figure 6 shows that As1-S3, As2-S2, and As2-S3 (As1-S1) intralayer distances increase above 0 (10) GPa, thus providing an explanation for the softening of several phonons in different pressure ranges. Notably, changes of the pressure coefficient of some experimental and theoretical Raman-active modes have been observed around $4 \mathrm{GPa}$ in $\alpha-\mathrm{As}_{2} \mathrm{~S}_{3}$, which could be attributed to the strong changes of the interatomic distances around these pressures. The changes can be clearly observed in the experimental $\mathrm{Bg}_{\mathrm{g}}{ }^{1}$ and $\mathrm{Ag}_{\mathrm{g}}{ }^{10}$ modes and in the theoretical $\mathrm{Ag}_{\mathrm{g}}{ }^{2}$, $B_{g}{ }^{1}, A_{g}{ }^{8}, A_{g}{ }^{9}, A_{g}{ }^{10}$ and $A_{g}{ }^{15}$ modes (see Figure 8(a)). They can also be observed in the theoretical $\mathrm{Bu}_{u^{1}}, \mathrm{~A}_{u^{2}}, \mathrm{~A}_{u^{3}}, \mathrm{~A}_{\mathrm{u}}{ }^{8}, \mathrm{~B}_{\mathrm{u}}{ }^{7}, \mathrm{~B}_{\mathrm{u}}{ }^{8}$ and $\mathrm{A}_{\mathrm{u}}{ }^{9}$ modes (see Figure $\mathbf{S 2 1}$ in the $\mathrm{SI}$ ), which do not evidence anticrossings between 0 and $6 \mathrm{GPa}$.

Figures $\mathbf{8 ( b )}$ and $\mathbf{S 2 1}$ show a notable softening of the theoretical vibrational modes of orpiment in the region between 18 and 26 GPa, where the pressure-induced IPT occurs according to our calculations. In order to probe the possibility of a second-order IPT occurring in $\alpha-\mathrm{As}_{2} \mathrm{~S}_{3}$, we have studied the phonon dispersion curves calculated at different pressure values, ranging up to 30 GPa (see Figures S22 and S23 in the SI), because a second-order IPT is related to the presence of a soft phonon mode according to Landau theory. Since we do not observe any soft mode up to
$30 \mathrm{GPa}$, we can conclude that the pressure-induced IPT occurring in $\alpha-\mathrm{As}_{2} \mathrm{~S}_{3}$ above $20 \mathrm{GPa}$ must be of order higher than 2 ; i.e. an IPT of electronic origin, such as the pressure-induced ETTs observed in other group-15 sesquichalcogenides [67].

In summary, HP-RS measurements and lattice-dynamics calculations in orpiment carried out up to $37 \mathrm{GPa}$ show that the lattice-dynamics of orpiment at HP is quite complex and has a large number of optical modes with negative pressure coefficient, related to the increase of some As-S bond distances, that cause a decrease of the phonon gap in orpiment under compression up to $20 \mathrm{GPa}$. Above this pressure, most phonons show a positive pressure coefficient, thus showing a complete change in the behavior of phonons of orpiment below and above $20 \mathrm{GPa}$; however, no soft modes going to zero frequency have been observed according to Landau theory so we can conclude that the IPT near $20 \mathrm{GPa}$ is not of second-order but of higher order; i.e. of electronic origin. Moreover, we have provided a tentative assignment of the Raman- and IR-active modes that can help in studying orpiment in ancient artworks using non-destructive techniques such as Raman and IR spectroscopy [39]. 


\section{ARTICLE}

Table 2 Theoretical (with vdW interactions) and experimental Raman-active mode frequencies and their respective pressure coefficients for $\alpha$-As $S_{2} S_{3}$ at room temperature, as fitted with equation $\omega(P)=\omega_{0}+\alpha \cdot P$. Experimental values from Refs. 23, 25 and $\mathbf{3 3}$ have been added for comparison. ${ }^{\text {a }}$ This work. ${ }^{b}$ Ref. 23. ${ }^{\mathrm{c}}$ Ref. 25. ${ }^{\mathrm{d}}$ Ref. $\mathbf{3 3} .{ }^{*}$ The pressure coefficient of these modes has been measured above $2 \mathrm{GPa}$.

\begin{tabular}{|c|c|c|c|c|c|c|}
\hline & Theoretical & & Experimental & & & \\
\hline Mode & $\omega_{0}\left(\mathrm{~cm}^{-1}\right)^{\mathrm{a}}$ & $\alpha\left(\frac{c m^{-1}}{G P a}\right) a$ & $\omega_{0}\left(\mathrm{~cm}^{-1}\right)^{\mathrm{a}}$ & $\alpha\left(\frac{c m^{-1}}{G P a}\right) \mathrm{a}$ & $\omega_{0}\left(\mathrm{~cm}^{-1}\right)$ & $\alpha\left(\frac{c m^{-1}}{G P a}\right)$ \\
\hline$A_{g}{ }^{1}$ & $27(1)$ & $5.1(3)$ & $26(1)$ & $6.32(37)$ & $25^{b}, 26^{c}$ & $9.0^{b}$ \\
\hline$A_{g}{ }^{2}$ & $38(1)$ & $4.5(2)$ & $37(1)$ & $4.9(3)$ & $36^{b}, 37^{c}$ & $7.2^{b}$ \\
\hline $\mathrm{Ag}^{3}$ & $59(1)$ & $4.1(2)$ & $62(1)$ & $3.7(1)$ & & \\
\hline $\mathrm{Bg}^{1}$ & $62(1)$ & $1.6(2)$ & & & $62^{b, c}$ & $5.0^{b}$ \\
\hline $\mathrm{Bg}^{2}$ & $68(1)$ & $10.3(4)$ & $69(3)$ & $10.5(8)$ & $69^{b}, 70^{c}$ & $11.0^{b}$ \\
\hline $\mathrm{Ag}^{4}$ & $70(1)$ & $5.9(3)$ & $70(3)$ & $8.1(3)$ & & \\
\hline $\mathrm{Bg}^{3}$ & $94(2)$ & $3.1(1)$ & $103(2)$ & $2.9(1)$ & & \\
\hline $\mathrm{Bg}^{4}$ & $103(2)$ & $2.1(5)$ & $106(2)$ & $2.8(1)$ & $107^{b}, 106^{c}$ & $4.9^{b}$ \\
\hline$A_{g}{ }^{2}$ & $124(2)$ & $7.7(7)$ & $138(5)$ & $5.1(4)$ & $136^{b}, 137^{c}, 135^{d}$ & $7.3^{b},-0.42^{d}$ \\
\hline $\mathrm{Bg}_{\mathrm{g}}{ }^{2}$ & $134(2)$ & $0.7(2)$ & $120(1)$ & $2.9(5)$ & & \\
\hline $\mathrm{Bg}^{6}$ & $135(2)$ & $3.6(1)$ & $144(1)$ & $3.9(2)$ & $145^{c}$ & \\
\hline $\mathrm{Bg}{ }^{7}$ & $152(3)$ & $3.7(2)$ & & & & \\
\hline$A_{g}{ }^{6}$ & $153(3)$ & $-4.6(7)$ & $155(2)$ & $-0.3(3)$ & $158^{c}$ & \\
\hline $\mathrm{Ag}{ }^{7}$ & $155(3)$ & $5.5(3)$ & $156(2)$ & $6.9(4)$ & $154^{b, c}, 153^{d}$ & $8.5^{b}, 0.24^{d}$ \\
\hline $\mathrm{Bg}_{\mathrm{g}}^{8}$ & $174(3)$ & $2.5(2)$ & $179(2)$ & $2.2(1)$ & $180^{c}, 177^{d}$ & $-2.22^{d}$ \\
\hline $\mathrm{Ag}_{\mathrm{g}}{ }^{2}$ & $176(3)$ & $1.2(1)$ & $185(2)$ & $2.3(1)$ & & \\
\hline $\mathrm{Bg}_{\mathrm{g}}{ }^{9}$ & $181(3)$ & $3.3(2)$ & $188(2)$ & $4.6(9)$ & $188^{c}$ & \\
\hline$A_{g}{ }^{9}$ & $196(3)$ & $1.3(1)$ & $202(1)$ & $2.5(2)$ & $202^{b}, 204^{c}, 201^{d}$ & $3.2^{b}$ \\
\hline$A_{g}{ }^{10}$ & $270(4)$ & $-3.1(2)$ & $291(1)$ & $-2.6(3)$ & $292^{b}, 293^{c}, 290^{d}$ & $-3.8^{b},-0.89^{d}$ \\
\hline $\mathrm{Bg}^{10}$ & $290(4)$ & $0.3(1)$ & $306(2)$ & $0.5(1)$ & $307^{c}, 308^{d}$ & $-0.2^{d}$ \\
\hline $\mathrm{Ag}_{\mathrm{g}}^{11}$ & $291(4)$ & $1.3(1)$ & $310(2)$ & $1.4(1)$ & $310^{b}, 312^{c}$ & $1.0^{\mathrm{b}}$ \\
\hline $\mathrm{Bg}^{11}$ & $305(4)$ & $-2.7(1)$ & $325(2)$ & $-3.5(4)$ & $326^{b, c}$ & $-4.6^{b}$ \\
\hline $\mathrm{Bg}^{12}$ & $329(5)$ & $-3.7(1)$ & 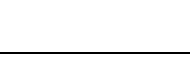 & 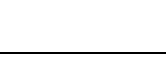 & $343^{c}$ & \\
\hline $\mathrm{Ag}_{\mathrm{g}}{ }^{12}$ & $341(5)$ & $1.0(5)$ & $356(4)$ & $-0.7(6)^{*}$ & $357^{b}, 360^{c}$ & $0.96^{b}$ \\
\hline $\mathrm{Ag}_{\mathrm{g}}{ }^{13}$ & $343(5)$ & $3.4(3)$ & $354(4)$ & $1.8(4)^{*}$ & $356^{c}$ & \\
\hline $\mathrm{Bg}^{13}$ & $345(5)$ & $3.1(1)$ & & & & \\
\hline $\mathrm{Ag}^{14}$ & $346(5)$ & $-0.5(1)$ & $353(4)$ & $4.1(6)^{*}$ & $354^{c}, 353^{d}$ & $-0.19^{d}$ \\
\hline $\mathrm{Bg}_{\mathrm{g}}{ }^{14}$ & $350(5)$ & $3.1(4)$ & $369(2)$ & $2.2(3)$ & $370^{c}$ & \\
\hline$A_{g}{ }^{15}$ & $369(5)$ & $2.4(1)$ & $383(2)$ & $2.2(2)$ & $383^{b}, 384^{c}, 380^{d}$ & $1.7^{b}, 0.47^{d}$ \\
\hline $\mathrm{Bg}^{15}$ & $385(5)$ & $-0.9(2)$ & $400(2)$ & $-0.7(3)$ & $401^{c}$ & \\
\hline
\end{tabular}




\section{ARTICLE}

\section{B.4 Electronic band structure calculations of $\alpha-A s_{2} S_{3}$ at HP}

To complete the picture of the behavior of orpiment at HP and in order to assess whether a pressure-induced ETT could be observed in orpiment, we have performed $a b$ initio electronic band-structure calculations of $\alpha-\mathrm{As}_{2} \mathrm{~S}_{3}$ at different pressures to identify possible changes of the band extrema that could be related to a pressure-induced ETT (see Figure 9). Our calculations show that orpiment is an indirect bandgap (1.7 eV) semiconductor at $0 \mathrm{GPa}$, with the valence band maximum (VBM) and conduction band minimum (CBM) being located along the high-symmetry segment of $Y-\Gamma$ and $\Gamma-Z$ directions, respectively. The value of the bandgap is clearly underestimated by our DFT calculations, since orpiment is known to have a bandgap around $2.7 \mathrm{eV}$ at room pressure $[26,31]$, but the structure of the electronic bands and the bandgap evolution with pressure are correctly described by this method. The bandgap energy value in our calculations is similar to the values of recent $a b$ initio calculations, however differing in the precise locations of the VBM and CBM $[33,55]$.
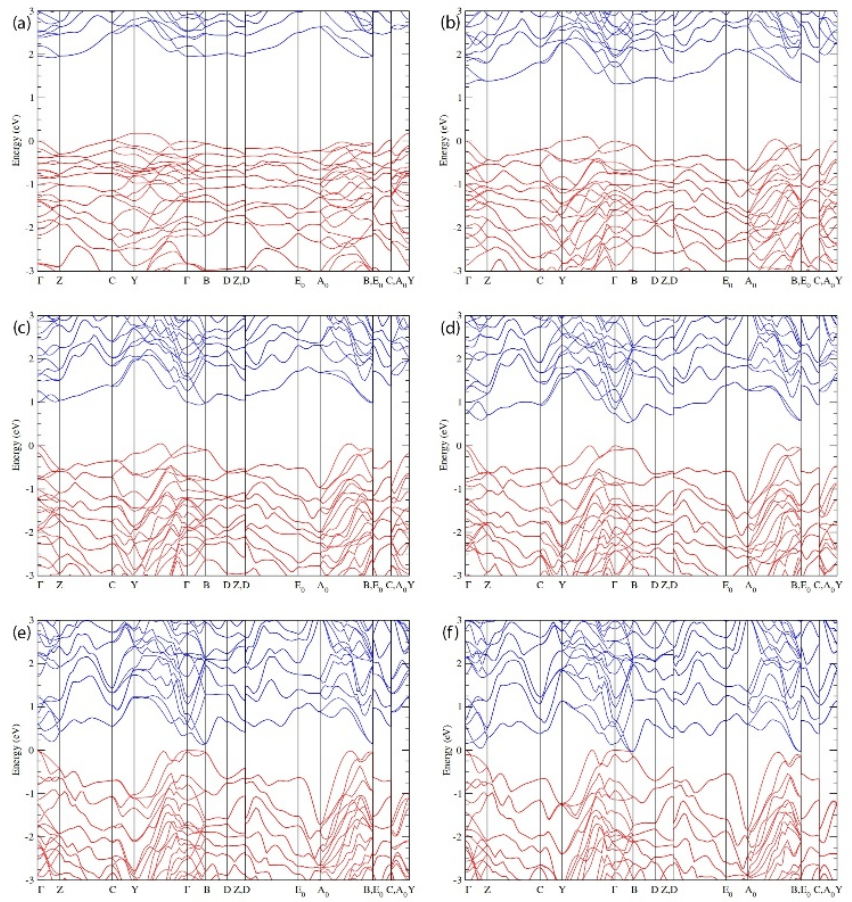

Figure 9. Calculated band structure of $\mathrm{As}_{2} \mathrm{~S}_{3}$ at different pressures: (a) 0 GPa, (b) $5 \mathrm{GPa}$, (c) $10 \mathrm{GPa}$, (d) $15 \mathrm{GPa}$, (e) $20 \mathrm{GPa}$, and (f) $30 \mathrm{GPa}$.

There are considerable changes in the VBM and CBM of $\alpha-\mathrm{As}_{2} \mathrm{~S}_{3}$ at HP. Both valence and conduction bands show a low dispersion across the $\mathrm{BZ}$ at $0 \mathrm{GPa}$, but a high dispersion above $20 \mathrm{GPa}$. The flat bands at $0 \mathrm{GPa}$ reflect the 2D character of orpiment, while the highly-dispersed bands above $20 \mathrm{GPa}$ reflect the 3D character of orpiment above this pressure. It can be observed that above $20 \mathrm{GPa}$, the VBM shifts to the $\Gamma$-point and along the $\Gamma-\mathrm{B}$ direction, whereas the CBM moves to the $\mathrm{B}$ point. Additionally, a variation of the VBM occurs between 20 and $30 \mathrm{GPa}$, with the VBM at $30 \mathrm{GPa}$ shifted along the highsymmetry $\Gamma-Y$ direction. Since we observe the crossing of an extremum through the Fermi level above $20 \mathrm{GPa}$, thus modifying of the topology of the Fermi-surface, we thus suggest a possible pressure-induced ETT above this pressure in $\alpha-\mathrm{As}_{2} \mathrm{~S}_{3}$. The calculated indirect bandgap exhibits a strong decrease at $\mathrm{HP}$ from $1.7 \mathrm{eV}$ at $0 \mathrm{GPa}$ to $0 \mathrm{eV}$ at $26 \mathrm{GPa}$ (see solid line in Figure 10). Therefore, our calculations provide evidence for a semiconducting-metallic transition in orpiment above $26 \mathrm{GPa}$. A recent experiment suggests that metallization in orpiment should occur above $42 \mathrm{GPa}$ [33]. If we assume that metallization occurs in the monoclinic $P 2_{1} / n$ phase around $42 \mathrm{GPa}$, the underestimation of the bandgap by our DFT calculations would be only $0.3 \mathrm{eV}$ (see long dashed line in Figure 10); however, if we take into account the value of the experimental bandgap at room pressure $(2.7 \mathrm{eV})$; i.e. assuming an underestimation of the bandgap of $1.0 \mathrm{eV}$, and shift the calculated bandgap to match the latter, the evolution of the bandgap (see short dashed line in Figure 10) agrees remarkably well with the behavior of the optical bandgap (symbols in Figure 10) [26,27]. In fact, the decrease of the electronic bandgap shows a theoretical pressure coefficient of $-0.16 \mathrm{eV} / \mathrm{GPa}$, which is in very good agreement with a previous experimental estimation of the optical bandgap pressure coefficient (-0.14 eV/GPa) [26,27,31]. Assuming the $1.0 \mathrm{eV}$ bandgap underestimation of our calculations, the extrapolation of the shifted calculated bandgap to $0 \mathrm{eV}$ yields a closure of the bandgap of the monoclinic $P 2_{1} / n$ phase at pressures that go beyond the studied range (above $50 \mathrm{GPa}$ ). This result suggests that the observed metallization at $42 \mathrm{GPa}$ [33] could be explained by the nonhydrostatic conditions in the sample during electric measurements or by a phase transition towards another phase. In this context, we have found a triclinic $P \overline{1}$ phase that is energetically more favorable than the $P 2_{1} / n$ phase above 40 GPa (see enthalpy vs pressure plot in Figure S24). This result suggests that it is possible the existence of a phase transition towards a metallic phase of $\mathrm{As}_{2} \mathrm{~S}_{3}$ above $40 \mathrm{GPa}$ in good agreement with Ref. 33. 
In summary, our calculations show that $\alpha-A s_{2} S_{3}$ is a semiconductor with an indirect bandgap ( $2.7 \mathrm{eV}$ at room pressure) that decreases at HP at an initial rate of $\sim-0.15$ $\mathrm{eV} / \mathrm{GPa}$ and becomes a metal well above $50 \mathrm{GPa}$. These features are in perfect agreement with already reported optical absorption measurements. Besides, we observe a clear change in the VBM above $20 \mathrm{GPa}$ that could lead to a pressure-induced ETT; i.e. an IPT of order $2^{1 / 2}$ according to Ehrenfest notation [74]. Furthermore, we propose that there could be a possible first-order phase transition in orpiment above $40 \mathrm{GPa}$ to a metallic phase that would explain the recent results on HP electrical measurements [33].

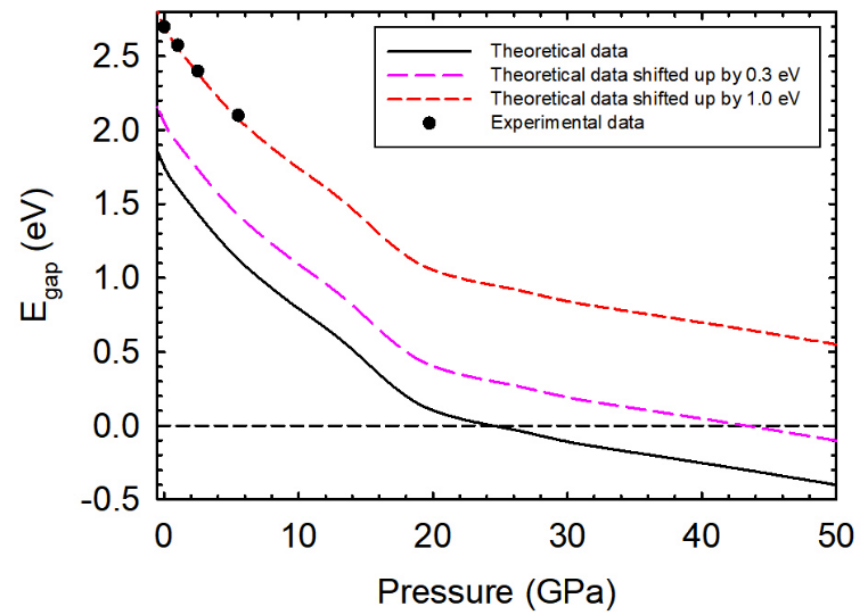

Figure 10. Pressure dependence of the energy bandgap of orpiment. Solid, long dashed and short dashed lines correspond to results of theoretical calculations including vdW interaction, theoretical calculations shifted by $0.3 \mathrm{eV}$ (to match the metallization observed above $42 \mathrm{GPa}$ in Ref. 33) and theoretical calculations shifted by $1.0 \mathrm{eV}$ (to match the optical bandgap at 0 GPa as in Refs. 26 and 27), respectively. Symbols correspond to experimental data from Refs. 26 and 27.

\section{B.5 Metavalent bonding in $\alpha-A s_{2} S_{3}$}

In this section, we will show that the results obtained so far for the pressure dependence of the structural, vibrational, optical and electric properties of $\alpha-\mathrm{As}_{2} \mathrm{~S}_{3}$ can be understood on the light of a special case of resonant bonding formalism [40-45]. This bonding has recently been termed as metavalent bonding in order to distinguish it from the resonant bonding occurring in benzene and graphite [44]. In particular, we will show that the HP behavior of orpiment between 0 and $20 \mathrm{GPa}$ can be considered as the process of change from p-type covalent bonding towards metavalent bonding at HP. Moreover, we will show that the concept of metavalent bonding could be extended to understand the pressure behavior of other group15 sesquichalcogenides and of trigonal Se and Te.

Metavalent bonding is a recently proposed new class of bonding formalism, mainly located between p-type covalent bonding and metallic bonding, which is characteristic of a new type of materials termed as "incipient metals" [44]. This kind of bonding occurs in materials where there is a deficiency of valence electrons in the unit cell to form a large number of bonds, such as in octahedrally-coordinated rocksalt-related structures as those found in GeTe, SnTe, PbSe, PbTe, Sb, Bi,
$\mathrm{Sb}_{2} \mathrm{Te}_{3}, \mathrm{Bi}_{2} \mathrm{Se}_{3}, \mathrm{Bi}_{2} \mathrm{Te}_{3}, \mathrm{AgSbTe}_{2}, \mathrm{AgBiSe}_{2}, \mathrm{AgBiTe}_{2}$, and $\mathrm{GeSb}_{2} \mathrm{Te}_{4}$ $[\mathbf{4 1}, \mathbf{4 4 , 4 5}]$. Under these circumstances, the few valence electrons available in the formula unit must be shared (resonate) between several bonds in order to provide the most symmetrical structure; i.e. with the largest number of similar bonds and with the largest possible cation coordination (highest packing), compatible with the valence electrons available and strain limitations, e.g. those imposed by stereoactive LEPs [7577].
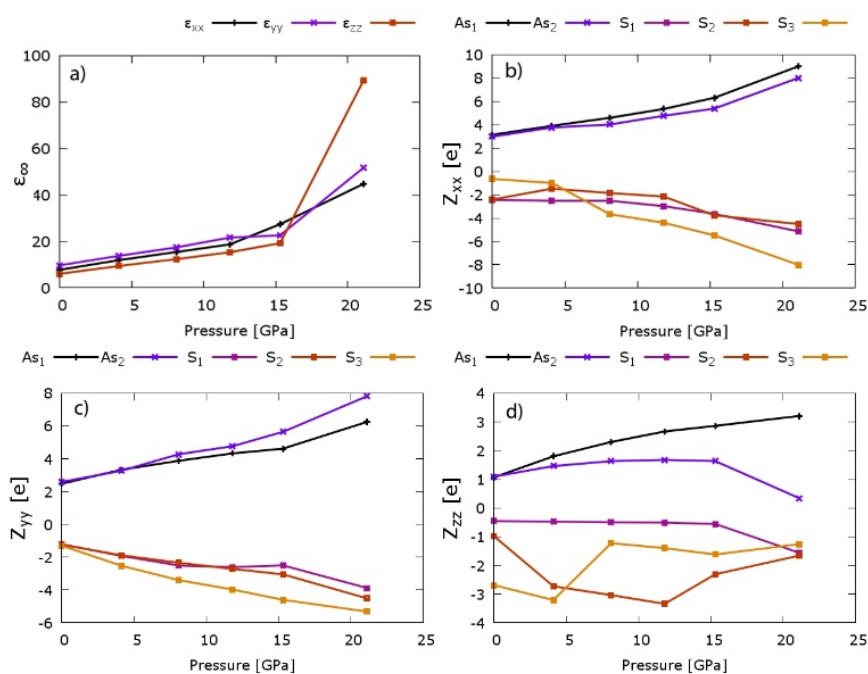

Figure 11. Pressure dependence of the static dielectric functions along the main crystallographic axes (a) and Born effective charges of the different As1, As2, S1, S2 and S3 atoms along the main crystallographic axes $(\mathbf{b}, \mathbf{c}, \mathbf{d})$.

The main characteristics of metavalent bonding are: i) a cation coordination much higher than that assumed with the 8-N rule; ii) high Born effective charges that are much larger than the valence of the atoms; iii) higher optical dielectric constants than typical covalent materials; iv) high mode Grüneisen parameters of phonons and lower wavenumbers of optical phonons than typical covalent materials, thus revealing a high lattice anharmonicity causing a very small thermal conductivity; and v) a moderately high electrical conductivity caused by a very small bandgap. All these features stem from the partial delocalization of electrons that are shared between several bonds. Due to these characteristics, materials featuring metavalent bonding have been named "incipient metals" because they show characteristics close to those of metals. In fact, they exhibit extraordinary properties that make them ideal candidates for phase-change materials, thermoelectric materials, and topological insulators.

Regarding orpiment, the equalization of the As-S intralayer distances above 18 (25) GPa according to our calculations without (with) considering vdW interactions is in agreement with the bonding variation nature of As-S bonds from covalent to metavalent [43-45]. To prove such fact, we have probed the bonding character of orpiment at different pressures; e.g. the electron localization function (ELF) along the seven nextneighbor As-S distances, the dielectric tensor, and the Born effective charges, as suggested in Ref. 44. 
The increase of the metavalent bonding in orpiment under compression can be traced by the increase of the theoretical dielectric constants and the Born effective charges (see Figure 11), as commented in Refs. $\mathbf{4 0}$ and $\mathbf{4 4}$. A strong increase in the absolute value of most of these magnitudes is observed between 0 and $20 \mathrm{GPa}$ [78]. The only Born effective charge component that does not show similar behavior within the range of 0 and $20 \mathrm{GPa}$ is the $Z_{z z}$ component, which is related to the $c$-axis, the direction of smallest change in bonding character because it is the direction of smaller compressibility (see Figure 5). These changes are a clear indication of the decrease (increase) of the covalent (metavalent) bonding of the layers of orpiment. Besides, the strong decrease of the optical bandgap with pressure and the metallization of orpiment well above 50 $\mathrm{GPa}$ is consistent with the metavalent bonding observed for chalcogenides $[\mathbf{4 4 , 4 5 ]}$. The rapid delocalization of the electron charge density over the plane of the layers caused by the steep increase of coordination of As atoms due to the strong compression of the chains mainly along the $a$-axis is the origin of the pronounced negative slope of the bandgap with increasing pressure.

Similarly to what was done in GeSe to study the pressure dependence of the Ge-Se bonds [43], we plot in Figure 12 the ELF values along the seven next-neighbor As1-S and As2-S distances obtained from our theoretical results at 0 and $25 \mathrm{GPa}$. At $0 \mathrm{GPa}$, both the As1 and As2 atoms have high ELF values (around 0.8 ) close to the center of the As-S bonds for each of the three bonds of each As atom (As1-S1, As1-S2, As1-S3, As2S1, As2-S2, and As2-S3); thus evidencing the threefold coordination of As1 and As2 atoms and the covalent bonding in orpiment at room pressure. However, at $25 \mathrm{GPa}$ there are five intralayer As2-S bonds with high ELF values (As2-S1 bond above 0.8 and two As2-S2 and two As2-S3 bonds above 0.6) showing fivefold coordination for As2 in orpiment. A similar situation occurs for the As1 atoms at $25 \mathrm{GPa}$, with five intralayer As1-S bonds with a relatively high ELF (As1-S2 bond near 0.8, two As1S1 above 0.7 and two As1-S3 above 0.5), but also with two interlayer As1-S2 bonds with an intermediate value of the ELF (slightly above 0.2 ), which can be considered to support $5+2$ coordination. Note that the typical metavalent bonding in the layer plane (ac-plane) of orpiment is clear for the As2 atoms, where all four intralayer bonds (As2-S2, As2-S3, As2-S4, and As2-S5) have the same ELF value because they have the same As2-S length. On the other hand, the character of As1-S bonds is more complex because the ELF values and lengths of all the intralayer bonds are not equal. Such behavior suggests that the intralayer metavalent bonding of As1 atoms is sacrificed by a larger coordination number taking into account $\mathrm{S}$ atoms of adjacent layers. Note that these $\mathrm{S}$ atoms are not linked to As2 atoms of the monoclinic structure even up to $50 \mathrm{GPa}$, what evidences the different behavior of As1 and As2 atoms on increasing pressure. The larger coordination of As1 atoms at HP is indicative of a tendency of As1 atoms to metallic bonding, which is usually characterized by a much larger effective coordination number than covalent and metavalent bondings $[44,45]$
The decrease of the bond force (ELF value) of some As-S bonds in orpiment is likely related to the length increase of those bonds (see Figure 6), because the charge of valence electrons in As atoms must progressively be redistributed among several equidistant As-S bonds within the context of metavalent bonding $[\mathbf{4 4 , 4 5}]$. Therefore, the charge at each of the short bonds at low pressure is redistributed between two equal bonds at HP leading to a smaller ELF for the initial short bonds at HP than room pressure. Such a feature is confirmed by the equal values of the ELF in As-S bonds of equal length at HP already commented. Therefore, the decrease of the ELF of the original covalent bonds at HP clearly shows the delocalization of electronic charge in these bonds to form metavalent bonds at HP.
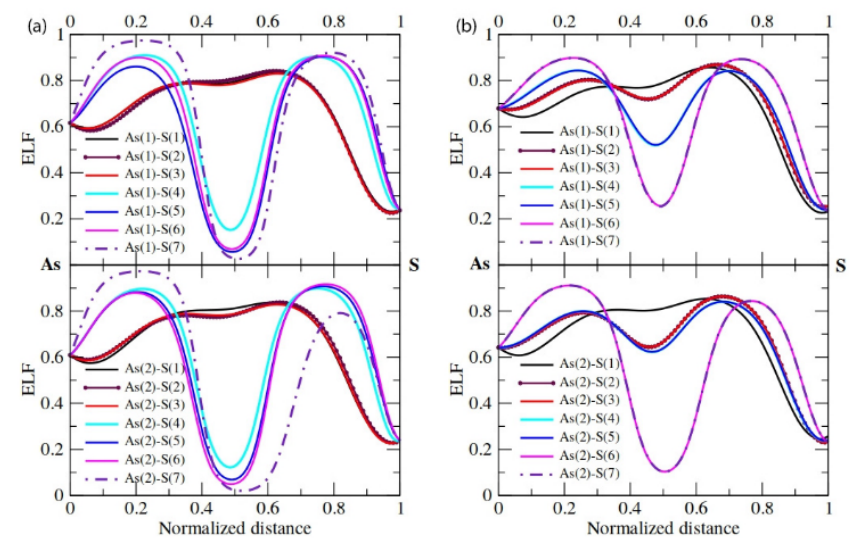

Figure 12. Pressure dependence of the ELF along the As1-S (top) and As2$\mathrm{S}$ (bottom) bonds at $0 \mathrm{GPa}$ (a) and $25 \mathrm{GPa}$ (b).

This bond enlargement and charge redistribution typical of the formation of metavalent bonds is expected to cause a decrease of some of the optical phonon frequencies. In particular, a softening of the transverse optical (TO) and longitudinal optical (LO) modes has been predicted in rocksalt compounds with metavalent bonding as compared to the same compound in absence of metavalent bonding $[\mathbf{4 1 , 4 2 ]}$. For a monoclinic compound, such as orpiment, the situation is more complex, because the TO and LO modes of the rocksalt structure are split into many components due to the decrease in symmetry. Therefore, we expect that many optical modes of orpiment will soften with increasing pressure due to the establishment of long-range interactions between neighboring atoms upon increasing the metavalent bonding at increasing pressure. In fact, Table 2 and Figure 8 show that one of the most characteristic modes that exhibit a negative pressure coefficient is the $\mathrm{Ag}_{\mathrm{g}}{ }^{10}$ mode (see atomic vibrations in Figure $\mathbf{S 1 1}$ in the $\mathrm{SI}$ ), that is experimentally observed at $291 \mathrm{~cm}^{-1}$ at room pressure. Other soft Raman- and IR-active modes can be observed in Figures $\mathbf{8}(\mathbf{b})$ and $\mathbf{S 2 1}$ in the $\mathrm{SI}$, respectively. Moreover, these last figures show a clear decrease of the phonon bandgap (between $A_{g}{ }^{9}$ and $A_{g}{ }^{10}$ and between $B_{u}{ }^{7}$ and $B_{u}{ }^{8}$ ) from 0 to $20 \mathrm{GPa}$. This decrease of the phonon bandgap is also expected in the context of metavalent bonding $[\mathbf{4 1}, \mathbf{4 2}, \mathbf{4 4}]$. Finally, we observed a considerable softening of several modes taking place between 18 and $26 \mathrm{GPa}$ and clear positive slopes of most vibrational modes above $26 \mathrm{GPa}$; i.e. once the bonding transition to 
metavalent bonding takes over. These features are in agreement with the development of metavalent bonding since low-frequency values of optical vibrational modes and positive slopes of all Raman-active and IR-active modes have been observed in $\mathrm{Bi}_{2} \mathrm{Se}_{3}, \mathrm{Sb}_{2} \mathrm{Te}_{3}$ and $\mathrm{Bi}_{2} \mathrm{Te}_{3}$, which are metavalent compounds at room pressure [67].

A closer look into the HP behavior of other chalcogenides evidences that the softening of optical modes at HP has been observed in a number of chalcogenides, thus suggesting that metavalent bonding is more common than expected at HP in these compounds. Several modes of the low-pressure trigonal phase of Se and Te exhibit a pronounced softening at HP. However, no softening has been found in the HP phases of these two elements $[\mathbf{7 9}, \mathbf{8 0}]$, as it happens in $\mathrm{Bi}_{2} \mathrm{Se}_{3}, \mathrm{Bi}_{2} \mathrm{Te}_{3}$ and $\mathrm{Sb}_{2} \mathrm{Te}_{3}$ [67]. This result is consistent with the recent confirmation of the metavalent bonding in $\mathrm{Bi}_{2} \mathrm{Se}_{3}, \mathrm{Bi}_{2} \mathrm{Te}_{3}$ and $\mathrm{Sb}_{2} \mathrm{Te}_{3}$ and $\beta-\mathrm{As}_{2} \mathrm{Te}_{3}$ at room conditions [81] and with the formation of metavalent bonding in Se and Te at HP [44]. Therefore, we expect a similar increase of dielectric constants and Born effective charges at HP in the low-pressure trigonal phase of $\mathrm{Se}$ and $\mathrm{Te}$, as it has been shown in orpiment and other compounds $[\mathbf{4 0 , 4 4 , 4 5 ] .}$

Finally, we want to emphasize that the analysis of the different group-15 sesquichalcogenides (Table S3 in the SI) reveals that their behavior at HP can also be understood in the framework of metavalent bonding. In particular, some of these materials possess metavalent bonding at room pressure [81] and our work shows that some others tend to a metavalent bonding at $\mathrm{HP}$. For instance, a similar situation to that of $\alpha-\mathrm{As}_{2} \mathrm{~S}_{3}$ is expected to occur for isostructural $\alpha-\mathrm{As}_{2} \mathrm{Se}_{3}$ and for $\beta-\mathrm{As}_{2} \mathrm{Se}_{3}$ at HP since both compounds feature a threefold coordination at room pressure. Unfortunately, there are almost no HP studies of these compounds. On the other hand, $\alpha-\mathrm{Sb}_{2} \mathrm{~S}_{3}, \alpha-\mathrm{Sb}_{2} \mathrm{Se}_{3}$ and $\alpha-\mathrm{Bi}_{2} \mathrm{~S}_{3}$, which crystallize in the orthorhombic Pnma phase, feature an average fourfold cation coordination, because one cation has threefold coordination and the other has fivefold coordination. Therefore, they show an intermediate cation coordination between the threefold coordination of $\alpha-\mathrm{As}_{2} \mathrm{~S}_{3}$ and the sixfold coordination of $\alpha-\mathrm{Sb}_{2} \mathrm{Te}_{3}$. They exhibit softening of some high-frequency modes [50-52]. In particular, softening of two high-frequency modes has been observed in $\alpha-\mathrm{Sb}_{2} \mathrm{~S}_{3}$ up to $12 \mathrm{GPa}$; pressure at which $\mathrm{Sb} 1$ and $\mathrm{Sb} 2$ can be considered to have almost a sevenfold coordination due to a pressureinduced metavalent bonding of both $\mathrm{Sb}$ atoms [52]. These compounds have been recently shown not to be metavalent at room pressure [81] becoming metavalent at HP according to our work.

An intermediate case between covalent and metavalent bonding is also that of $\alpha-\mathrm{As}_{2} \mathrm{Te}_{3}$, which shows an average cation coordination of 5.5 , because one cation has $3+2$ coordination and the other has $3+3$ coordination. Therefore, it shows an intermediate coordination between the fivefold coordination of $\alpha-\mathrm{Sb}_{2} \mathrm{Se}_{3}$ and the sixfold coordination of $\alpha-\mathrm{Sb}_{2} \mathrm{Te}_{3}$. Consequently, $\alpha-\mathrm{As}_{2} \mathrm{Te}_{3}$ shows almost no soft phonons. Only a few of high-frequency phonons show a soft behavior with almost negligible pressure coefficient between 0 and $4 \mathrm{GPa}$ and a notable hardening above this pressure [53]. An inspection of the evolution of the As-Te distances in this pressure range shows that the As1 (As2) coordination changes from almost five (six) at room pressure to real six (six) above $4 \mathrm{GPa}$. Therefore, the changes observed in the pressure coefficients of zonecenter vibrational modes in $\alpha-\mathrm{As}_{2} \mathrm{Te}_{3}$ are indicative of a change of bonding type from an almost metavalent bonding inside the layers to a full metavalent bonding that takes into account also interactions between the layers. Finally, $\beta-\mathrm{As}_{2} \mathrm{Te}_{3}$ with $R \overline{3} m$ symmetry and sixfold coordination has been confirmed to show metavalent bonding [81] and is expected to show no soft modes, as $\alpha-\mathrm{Bi}_{2} \mathrm{Se}_{3}, \alpha-\mathrm{Bi}_{2} \mathrm{Te}_{3}$ and $\alpha-\mathrm{Sb}_{2} \mathrm{Te}_{3}$ [67].

It must be noted that a notable decrease of the bandgap with pressure has also been obtained in DFT calculations for $\alpha-\mathrm{Sb}_{2} \mathrm{~S}_{3}$, $\alpha-\mathrm{Sb}_{2} \mathrm{Se}_{3}$ and $\alpha-\mathrm{Bi}_{2} \mathrm{~S}_{3}[52]$, and at a smaller rate also for $\alpha-\mathrm{As}_{2} \mathrm{Te}_{3}$ [67] and $\mathrm{Bi}_{2} \mathrm{Te}_{3}$ [82]. In fact, we have observed a metallization around $26 \mathrm{GPa}$ in our DFT calculations for $\alpha-\mathrm{As}_{2} \mathrm{~S}_{3}$, similar to the metallization found around $4 \mathrm{GPa}$ for $\alpha-\mathrm{As}_{2} \mathrm{Te}_{3}$ and above 12 $\mathrm{GPa}$ for $\alpha-\mathrm{Sb}_{2} \mathrm{~S}_{3}$. Therefore, it is expected that a similar increase of dielectric tensors and the Born effective charges at HP occurs in these group-15 sesquichalcogenides, thus supporting the occurrence of metavalent bonding in these compounds at HP.

\section{Experimental and Theoretical description}

\section{C.1 Experimental details}

Almost pure mineral orpiment from Hunan province (China) was used in the present study. Angle-dispersive powder HP-XRD experiments at room temperature up to $25 \mathrm{GPa}$ were conducted in a membrane-type diamond anvil cell (DAC) at the BL04-MSPD beamline of ALBA synchrotron. Incident monochromatic beam with wavelength of $0.4246 \AA$ was focused to $20 \times 20 \mu \mathrm{m}^{2}$ using a pinhole of $50 \mu \mathrm{m}$ to cut the $\mathrm{x}$-ray beam tail [83]. Images covering a $2 \theta$ range up to $18^{\circ}$ were collected using a SX165 CCD located at $240 \mathrm{~mm}$ from the sample. Onedimensional diffraction profiles of intensity as a function of $2 \theta$ were obtained by integration of the observed intensities with the Fit2D software [84]. Le Bail refinements were carried out with GSAS package software for synchrotron measurements $[\mathbf{8 5}, \mathbf{8 6}]$. Interatomic distances were obtained with the Visualization for Electronic and Structural Analysis (VESTA) software [87]. The equation of state (EoS) of copper [88], which was mixed with the sample powder, was used for pressure calibration.

Room-temperature unpolarized HP-RS measurements up to 14.5 GPa were carried out with a Horiba Jobin Yvon LabRAM HR spectrometer equipped with a thermoelectrically cooled multichannel CCD detector. RS measurements with a spectral resolution better than $2 \mathrm{~cm}^{-1}$ were excited using the $632.8 \mathrm{~nm}$ line of a He:Ne laser with a power below $1 \mathrm{~mW}$ in order to avoid laser heating of the sample. The use of an edge filter limited the observation of Raman-active modes below $40 \mathrm{~cm}^{-1}$. Pressure was determined with the ruby fluorescence method [89]. In both HP-XRD and HP-RS measurements, a methanol-ethanol (4:1 ratio) mixture was used as a pressure-transmitting medium with quasi-hydrostatic conditions up to $10 \mathrm{GPa}$ and deviatoric stresses are within the experimental uncertainty of this soft material up to $25 \mathrm{GPa}[\mathbf{9 0 , 9 1 ]}$. 


\section{C.2 Theoretical details}

$A b$ initio calculations within the density functional theory (DFT) [92] were performed to compute the electronic and structural properties of $\alpha-A s_{2} S_{3}$ by using the plane-wave Vienna $A b$ initio Simulation Package (VASP) $[\mathbf{9 3}, \mathbf{9 4}]$. The projector-augmented wave scheme (PAW) [95] was considered to treat the valence and the semi-core states, more explicitly by including the $4 \mathrm{~s}$ and $4 p$ outermost electrons of As and the $3 s$ and $3 p$ electrons of $S$ as valence electrons, with the remaining of the electrons being considered frozen at the core. In this work, the generalized gradient approximation with the Perdew-Burke-Ernzerhof (PBE) [96] parametrization was used for the exchange and correlation energy, after evaluating calculations performed also with the PBEsol modified version [97] in Ref. 35. The plane-wave kineticenergy cutoff was defined with $360 \mathrm{eV}$, which together with the use of a dense Monkhorst-Pack grid [98] with a $6 \times 4 \times 2$ k-point reciprocal space sampling mesh, ensured a convergence of the total energy around $1 \mathrm{meV}$ with deviations of the stress tensor from a diagonal hydrostatic form of less than $1 \mathrm{kbar}(0.1 \mathrm{GPa}$ ). The vdW corrections to the total energy were taken into account within DFT by using the D2 method [99]. Electronic band-structure calculations were carried out at different pressures along selected high-symmetry k-points on the first Brillouin-zone (BZ). Additionally, analysis of the electron density topology and of the electron localization function (ELF) were accomplished with the VESTA program employing data from the VASP code $[100,101]$.

Lattice-dynamics calculations were performed at the zone center ( $\Gamma$-point) and along high-symmetry segments of the BZ as a function of pressure, by using the direct-force constant approach $[\mathbf{1 0 2}, \mathbf{1 0 3}]$. The separate calculations of the forces, which result from a fixed displacement away from equilibrium of the atoms in the primitive cell, necessary to the construction of the dynamical matrix at the $\Gamma$-point of the $B Z$ were achieved with VASP. The diagonalization of the dynamical matrix provides the normal-mode frequencies and allows identifying the irreducible representations and the character of the vibrational phonon modes at the $\Gamma$-point.

\section{Conclusions}

The monoclinic structure of orpiment $\left(\alpha-\mathrm{As}_{2} \mathrm{~S}_{3}\right)$ at room conditions is composed of layers formed by AsS spiral chains, where the As atoms have threefold coordination. We have studied the structural, vibrational, and electronic properties of orpiment at room temperature and HP and have found that orpiment undergoes a strong compression up to $20 \mathrm{GPa}$, followed by an IPT of electronic origin, that leads to a coordination of As which is higher than five above that pressure. The most striking feature of the pressure-induced IPT is that several As-S bond distances become equal above $20 \mathrm{GPa}$.

The lattice dynamics of orpiment has been studied and all the rigid layer modes and the main inter-chain and intra-chain modes have been fully described. Our measurements and calculations evidence the softening of many vibrational modes and the decrease of the phonon gap (it closes above $20 \mathrm{GPa}$ ) at HP. Moreover, our calculations confirm the metallization of orpiment above $26 \mathrm{GPa}$ due to the strong decrease of the optical bandgap, thus supporting metallization in laboratory under hydrostatic conditions well above $50 \mathrm{GPa}$ when taking into consideration the underestimation of the energy bandgap in DFT calculations. All these changes are related to the formation of metavalent bonding occurring in orpiment above $20 \mathrm{GPa}$ due to the delocalization of electronic clouds, mainly in the ac-plane of the layers, leading to an increase of cation coordination (more than fivefold) without the need for a firstorder structural phase transition as in Se and Te, and similar to GeSe upon the Pnma to $\mathrm{Cmcm}$ phase transition [43].

On top of that, we have suggested that metavalent bonding already occurs in other group-15 sesquichalcogenides at room pressure or at HP. Full metavalent bonding occurs in topological insulators and good thermoelectric materials as $\alpha-\mathrm{Bi}_{2} \mathrm{Se}_{3}, \alpha-$ $\mathrm{Bi}_{2} \mathrm{Te}_{3}$ and $\alpha-\mathrm{Sb}_{2} \mathrm{Te}_{3}$ with tetradymite layered structure that feature a sixfold cation coordination. In these compounds, transformation from metavalent to metallic bonding occurs at HP (still not proved for $\beta-\mathrm{As}_{2} \mathrm{Te}_{3}$ ) after a first-order phase transition to a structure with a cation coordination larger than the original sixfold one. On the other hand, a partial metavalent bonding occurs in $\alpha-\mathrm{Bi}_{2} \mathrm{~S}_{3}, \alpha-\mathrm{Sb}_{2} \mathrm{~S}_{3}$ and $\alpha-\mathrm{Sb}_{2} \mathrm{Se}_{3}$, with an orthorhombic Pnma structure, and in $\alpha-\mathrm{As}_{2} \mathrm{Te}_{3}$. These compounds show an intermediate coordination between three and six. In all these compounds, pressure increases cation coordination leading to a considerable bandgap reduction and partial phonon softening consistent with metavalent bonding at HP. Finally, we predict that a similar situation than for $\mathrm{As}_{2} \mathrm{~S}_{3}$ is expected for $\mathrm{As}_{2} \mathrm{Se}_{3}$ polymorphs and also in the low-pressure trigonal Se and Te at HP. These compounds featuring threefold coordination at room pressure are expected to exhibit metavalent bonding at HP.

In summary, our present results on $\alpha-\mathrm{As}_{2} \mathrm{~S}_{3}$ confirm that pressure is able to tune the metavalent bonding in group-15 sesquichalcogenides, such as occurs for orpiment, thus turning common semiconductors into "incipient metals" with promising phase-change, thermoelectric and topological insulating properties at extreme conditions. Since $\mathrm{Sb}_{2} \mathrm{Te}_{3}$ and $\mathrm{Bi}_{2} \mathrm{Te}_{3}$ are topological insulators and two of the best known thermoelectric materials at room conditions due to metavalent bonding [81], this work paves the way to design new group-15 sesquichalcogenides and related compounds with thermoelectric or topological insulating properties both at room pressure and at extreme conditions.

\section{Conflicts of interest}

There are no conflicts to declare

\section{Acknowledgements}

Authors thank the financial support from Spanish Ministerio de Economia y Competitividad (MINECO) through MAT2016- 
75586-C4-2/3-P and FIS2017-83295-P and from Generalitat Valenciana under project PROMETEO/2018/123-EFIMAT. ELDS acknowledges the European Union Horizon 2020 research and innovation programme under Marie Sklodowska-Curie for grant agreement No. 785789-COMEX. JAS also acknowledges Ramón y Cajal program for funding support through RYC-2015-17482. AM, SR and ELDS thank interesting discussions with J. ContrerasGarcía who taught them how to analyze the ELF. Finally, authors thank ALBA Light Source for beam allocation at beamline MSPD (Experiment No. 2013110699) and acknowledge computing time provided by MALTA-Cluster and Red Española de Supercomputación (RES) through computer resources at MareNostrum with technical support provided by the Barcelona Supercomputing Center (QCM-2018-3-0032).

\section{Notes and references}

1 J. D. Smith, J. C. Bailar, H. J. Emeléus, R. Nyholm, The Chemistry of Arsenic, Antimony and Bismuth, Pergamon Texts in Inorganic Chemistry, vol. 2 (1973).

2 Pliny the Elder, Naturalis Historia, Chapter 22, ed. by J. Bostock, M.D., F.R.S. H.T. Riley, Esq., B.A. London (Taylor and Francis, London, 1855).

3 E. W. Fitzhugh, Orpiment and Realgar, in Artists' Pigments, A Handbook of Their History and Characteristics, vol. 3 (Oxford University Press, 1997), p. 47-80.

4 F.C.J. Spurrell, Archaeological J. 1895, 52, 222-239.

5 L. Burgio and R. J. H. Clark, J. Raman Spectrosc. 2000, 31, 395-401.

6 S. Waxman and K. C. Anderson, Oncologist 2001, 6 (Suppl 2), 3-10.

7 W. Ding, Y. Tong, X. Zhang, M. Pan, and S. Chen, Sci. Rep. 2016, 6, 19793.

8 J. Heo, and W.J. Chung, Rare-earth-doped chalcogenide glass for lasers and amplifiers, in Chalcogenide Glasses: Preparation, Properties and Applications (Woodhead Publishing, 2014), pp. 347-380.

9 D. W. Hewak, N. I. Zheludev, and K.F. MacDonald, Controlling light on the nanoscale with chalcogenide thin films, in Chalcogenide Glasses: Preparation, Properties and Applications (Woodhead Publishing, 2014), pp. 471-508.

10 N. Morimoto, Miner. J. 1954, 1, 160-169.

11 D. J. E. Mullen and W. Nowacki, Z. Kristall. 1972, 136, 48-65.

12 A. R. Kampf, R.T. Downs, R. M. Housley, R. A. Jenkins, J. Hyršl, Mineral Mag. 2011, 75, 2857-2867.

13 G. V. Gibbs, A. F. Wallace, R. Zallen, R. T. Downs, N. L. Ross, D. F. Cox, K. M. Rosso, J. Phys. Chem. A 2010, 114, 6550-6557.

14 H.F. Cheng, Y. Zhou, and R. L. Frost, Spectros. Lett. 2017, 50, 23-29.

15 S. P. S. Porto, D. L. Wood, J. Opt. Soc. Am. 1962, 52, 251-252.

16 A. Weber, S. P. S. Porto, J. Opt. Soc. Am. 1965, 55, 1033-1034.

17 A. T. Ward, J. Phys. Chem. 1968, 72, 4133-4139.

18 R. Forneris, Am. Miner. 1969, 54, 1062-1074.

19 W. Scheuermann, G. J. Ritter, Z. Naturforsch. 1969, A24 408-411.

20 J. M. Mathieu, H. Poulet, Bull. Soc. Fr. Mineral. Cristall. 1970, 93, 532-535.

21 R. Zallen, M. Slade, A. T. Ward, Phys. Rev. B 1971, 3, 4257-4273.

22 R. Zallen, M. Slade, Phys. Rev. B 1974, 9, 1627-1637.

23 R. Zallen, Phys. Rev. B 1974, 9, 4485-4496.

24 A.P. DeFonzo, J. Tauc, Phys. Rev. B 1978, 18, 6957-6972.

25 C. Razzetti, P. P. Lottici, Solid State Commun. 1979, 29 361-364.
26 J. M. Besson, J. Cernogora, R. Zallen, Phys. Rev. B 1980, 22 3866-3876.

27 J. M. Besson, J. Cernogora, M. L. Slade, B. A. Weinstein, R. Zallen, Physica B 1981, 105, 319-323.

28 R. L. Frost, W. N. Martens, J. T. Kloprogge, N. Jb. Miner. Mh. Jg. 2002, 10, 469- 480.

29 S. Mamedov, N. Drichko, MRS Advances 2018, 3, 385-390.

30 J. P. Itié, A. Polian, M. Grimsditch, S. Susman, Jpn. J. Appl. Phys. 1992, 32, 719-721.

31 R. Zallen, High Press. Res. 2004, 24, 117-118.

32 N. B. Bolotina, V. V. Brazhkin, T. I. Dyuzheva, Y. Katayama, L. F. Kulikova, L. V. Lityagina, N. A. Nikolaev, JETP Lett. 2013, 98 539-543.

33 K. X. Liu, L. D. Dai, H. P. Li, H. Y. Hu, L. F. Yang, C. Pu, M. L. Hong, P.F. Liu, Materials 2019, 12, 784

34 E. A. Kravchenko, A. V. Timofeeva, G. Z. Virogradova, J. Mol. Str. 1980, 58, 253-262.

35 S. Radescu, A. Mujica, P. Rodríguez-Hernández, A. Muñoz, J. Ibáñez, J. A. Sans, V. P. Cuenca-Gotor, F. J. Manjón, J. Phys.: Conf. Series 2017, 950, 042018.

36 M. Šiškins, M. Lee, F. Alijani, M.R. van Blankenstein, D. Davidovikj, H. S. J. van der Zant, and P.G. Steeneken, ACS Nano 2019, 13, 10845-10851.

37 Z. Bao and X.D. Chen, Adv. Mater. 2016, 28, 4177-4179.

38 J.H. Koo, D. C. Kim, H. J. Shim, T.-H. Kim, and D.-H. Kim, Adv. Funct. Mater. 2018, 28, 1801834.

39 M. A. García-Bucio, M.Á. Maynez-Rojas, E. CasanovaGonzález, J. J. Cárcamo-Vega, J. L. Ruvalcaba-Sil, A. Mitrani, J. Raman Spectrosc. 2019, 50, 1546-1554.

40 K. Shportko, S. Kremers, M. Woda, D. Lencer, J. Robertson, M. Wuttig, Nat. Mat. 2008, 7, 653-658.

41 S. Lee, K. Esfarjani, T. F. Luo, J. W. Zhou, Z. T. Tian, G. Chen, Nat. Comm. 2014, 5, 3525.

42 C. W. Li, J. Hong, A. F. May, D. Bansal, S. Chi, T. Hong, G. Ehlers, O. Delaire, Nat. Phys. 2015, 11, 1063-1069.

$43 \mathrm{M}$. Xu, S. Jakobs, R. Mazzarello, J.-Y. Cho, Z. Yang, H. Hollermann, D. S. Shang, X. S. Miao, Z. H. Yu, L. Wang, M. Wuttig, J. Phys. Chem. C 2017, 121, 25447-25454.

44 M. Wuttig, W. L. Deriger, X. Gonze, C. Bichara, J.-Y. Raty, Adv. Mat. 2018, 30, 1803777.

45 J.-Y. Raty, M. Schumacher, P. Golub, V. L. Deringer, C. Gatti, M. Wuttig, Adv. Mater. 2018, 31, 1806280.

46 C. Svensson, Acta. Cryst. B 1974, 30, 458-461.

47 A. C. Stergiou, P. J. Rentzeperis, Z. Kristall. 1985, 173, 185-191.

48 F. Pertlik, Monatshefte für Chemie 1978, 109, 277-282.

49 A. Brown, S. Rundqvist, Acta Cryst. 1965, 19, 684-685.

50 I. Efthimiopoulos, J. M. Zhang, M. Kucway, C. Y. Park, R. C. Ewing, Y. Wang, Sci. Rep. 2013, 3, 2665-2672.

51 I. Efthimiopoulos, J. Kemichick, X. Zhou, S. V. Khare, D. Ikuta, Y. Wang, J. Phys. Chem. A 2014, 118, 1713-1720.

52 J. Ibañez, J. A. Sans, C. Popescu, J. López-Vidrier, J. J. ElviraBetanzos, V. P. Cuenca-Gotor, O. Gomis, F. J. Manjón, P. Rodríguez-Hernández, A. Muñoz, J. Phys. Chem. C 2016, 120, 10547-10558.

53 V. P. Cuenca-Gotor, J. A. Sans, J. Ibáñez, C. Popescu, O. Gomis, R. Vilaplana, F. J. Manjón, A. Leonardo, E. Sagasta, A. SuárezAlcubilla, I. G. Gurtubay, M. Mollar, A. Bergara, J. Phys. Chem. C 2016, 120, 19340-19352.

54 A. Walsh, D. J. Payne, R. G. Egdell, G.W. Watson, Chem. Soc Rev. 2011, 40, 4455-4463.

55 P. Srivastava, H. Singh Mund, Y. Sharma, Physica B 2011, 406, 3083-3088.

56 E. Kroumova, M.I. Arroyo, J.M. Perez-Mato, A. Kirov, C. Capillas, S. Ivantchev, H. Wondratschek, Phase Transitions 2003, 76, 155-170.

57 P. Canepa, R. M. Hanson, P. Ugliengo, M. Alfredsson, J. Appl. Cryst. 2011, 44, 225-229. 
58 H. Siebert, Z. Anorg. Allg. Chemie 1954, 275, 225-240.

59 F. Birch, J. Appl. Phys. 1938, 9, 279-288.

60 P. A. Guńka, M. Dranka, M. Hanfland, K. F. Dziubek, A. Katrusiak, J. Zachara, Cryst. Growth Des. 2015, 15, 3950-3954.

61 S. Haussühl, Physical Properties of Crystals. An Introduction (Wiley-VCH, 2007).

62 R. J. Angel, http://www.rossangel.com/text_strain.htm

63 S. Minomura, K. Aoki, N. Koshizuka, T. Tsushima, HighPressure Science and Technology (Springer, 1979), p. 435.

64 A. K. Bandyophadhay, D. B. Singh, Pramana 1999, 52, 303-319.

65 Y. A. Sorb, V. Rajaji, P. S. Malavi, U. Subbarao, P. Halappa, S. C. Peter, S. Karmakar, C. Narayana, J. Phys.: Condens. Mat. 2016 28, 015602.

66 I. Efthimiopoulos, C. Buchan, Y. Wang, Sci. Rep. 2016, 6, 24246.

67 F. J. Manjón, R. Vilaplana, O. Gomis, E. Perez-Gonzalez, D. Santamaria-Perez, V. Marin-Borras, A. Segura, J. Gonzalez, P. Rodriguez-Hernandez, A. Muñoz, Phys. Status Solidi B 2013, 250, 669-676.

68 J. A. Sans, F. J. Manjón, A. L. J. Pereira, R. Vilaplana, O. Gomis, A. Segura, A. Muñoz, P. Rodríguez-Hernández, C. Popescu, C. Drasar, P. Ruleova, Phys. Rev. B 2016, 93, 024110.

69 A. L. J. Pereira, D. Santamaría-Pérez, J. Ruiz-Fuertes, F. J. Manjón, V. P. Cuenca-Gotor, R. Vilaplana, O. Gomis, C. Popescu, A. Muñoz, P. Rodríguez-Hernández, A. Segura, L. Gracia, A. Beltrán, P. Ruleova, C. Drasar, J. A. Sans, J. Phys. Chem. C 2018, 122, 8853-8867.

70 O. Degtyareva, E. R. Hernández, J. Serrano, M. Somayazulu, H.-K. Mao, E. Gregoryanz, R. J. Hemley, J. Chem. Phys. 2007, 126, 084503.

71 W. Richter, J. B. Renucci, M. Cardona, Phys. Status Solidi B 1973, 56, 223-229.

72 K. Aoki, O. Shimomura, S. Minomura, N. Koshizuka, T. Tsushima, J. Phys. Soc. Jpn. 1980, 48, 906-911.

73 G. Lucogsky, Phys. Stat. Sol. B 1972, 49, 633-641.

74 I. M. Lifshitz, Sov. Phys. JETP 1960, 11, 1130.

75 I. D. Brown, Acta Cryst. B 1988, 44, 545-553.

76 M. Dudev, J. Wang, T. Dudev, C. Lim, J. Phys. Chem. B 2006, 110, 1889-1895.

77 I. D. Brown, American Mineralogist 2016, 101, 531-539.

78 No value is obtained at higher pressure since the metallization of the monoclinic structure above $25 \mathrm{GPa}$ in our calculations yields unreasonably high values of these parameters.

79 L. Properzi, A. Polian, P. Munsch, A. Di Cicco, High Pressure Research 2013, 33, 35-39.

80 C. Marini, D. Chermisi, M. Lavagnini, D. Di Castro, C. Petrillo, L. Degiorgi, S. Scandolo, P. Postorino, Physical Review B 2012, 86, 064103.

81 Y. Chen, O. Cojocaru-Mirédin, J. Keutgen, Y. Yu, M. Küpers, M. Shumacher, P. Golub, J.-Y. Raty, R. Dronskowski, M. Wuttig, Adv. Mat. 2019, 31, 1904316.

82 R. Vilaplana, O. Gomis, F. J. Manjón, A. Segura, E. PérezGonzález, P. Rodríguez-Hernández, A. Muñoz, J. González, V. Marín-Borrás, V. Muñoz-Sanjosé, C. Drasar, V. Kucek, Phys. Rev. B 2011, 84, 104112.

83 F. Fauth, I. Peral, C. Popescu, M. Knapp, Powder Diffr. 2013, 28, S360-S370.

84 A. P. Hammersley, S. O. Svensson, M. Hanfland, A. N. Fitch, D. Hausermann, High Pres. Res. 1996, 14, 235-248.

85 A. C. Larson, R. B. von Dreele, LANL Report 2004, 86, 748.

86 B. H. Toby, J. Appl. Crystallogr. 2001, 34, 210-213.

87 K. Momma, F. Izumi, J. Appl. Crystallogr. 2011, 44, 1272-1276.

88 A. Dewaele, P. Loubeyre, M. Mezouar, Phys. Rev. B 2004, 70 094112.

89 H. K. Mao, J. Xu, P. M. Bell, J. Geophys. Res. 1986, 91 4673-4676.
90 G. J. Piermarini, S. Block, J.D. Barnett, J. Appl. Phys. 1973, 44, 5377-5382.

91 S. Klotz, J.-C. Chervin, P. Munsch, G. Le Marchand, J. Phys. D: Appl. Phys. 2009, 42, 075413.

92 P. Hohenberg, W. Kohn, Phys. Rev. 1964, 136, B864-B871.

93 G. Kresse, J. Hafner, Phys. Rev. B 1993, 47, 558-561.

94 G. Kresse, J. Furthmüller, Comput. Mat. Sci. 1996, 6, 15-50.

95 P. E. Blöchl, Phys. Rev. B 1994, 50, 17953-17979.

96 J. P. Perdew, K. Burke, M. Ernzerhof, Phys. Rev. Lett. 1996, 77, 3865-3868.

97 J. P. Perdew, A. Ruzsinszky, G. I. Csonka, O. A. Vydrov, G. E. Scuseria, L. A. Constantin, X. Zhou, K. Burke, Phys. Rev. Lett. 2008, 100, 136406 .

98 H. J. Monkhorst, J. D. Pack, Phys. Rev. B 1976, 13, 5188-5192.

99 S. Grimme, J. Comp. Chem. 2006, 27, 1787-1799.

100 J. Contreras-García, A. Martin Pendás, B. Silvi, J.M. Recio, J. Phys. Chem. Solids 2008, 69, 2204-2207.

101 J. Contreras-García, B. Silvi, A. Martín Pendás, J.M. Recio, J. Chem. Theory Comput. 2009, 5, 164-173.

102 K. Parlinski, Z. Q. Li, Y. Kawazoe, Phys. Rev. Lett. 1997, 78, 4063-4066.

103 D. Alfè, Comp. Phys. Commun. 2009, 180, 2622-2633. 